\title{
Gas and dust emission in the Lupus globular filaments GF 17 and GF $20^{\star}$
}

\author{
M. C. Moreira and J. L. Yun \\ Centro de Astronomia e Astrofísica da Universidade de Lisboa, Tapada da Ajuda, 1349-018 Lisboa, Portugal
}

Received 2 May 2001 / Accepted 25 October 2001

\begin{abstract}
We present large-scale millimeter molecular line observations as well as IRAS co-added images of the globular filaments GF 17 and GF 20 in the southern constellation of Lupus. A comparison is made of the extended far-infrared emission detected by IRAS at 60 and $100 \mu \mathrm{m}$ and the $\mathrm{CO}(1-0),{ }^{13} \mathrm{CO}(1-0)$, and $\mathrm{C}^{18} \mathrm{O}(1-0)$ emission in GF 17 and GF 20. Based on the far-infrared emission estimates of the dust temperature, optical depth and visual extinction are derived. We find a correlation between the measured dispersion in our extinction determinations and the extinction toward the clouds, which is very similar to that found for other clouds, and interpret this as evidence that the cloud edges are characterized by a smooth density gradient. We find a remarkably good agreement between our $100 \mu \mathrm{m}$ optical depth images and the ${ }^{13} \mathrm{CO}$ integrated emission maps. The dust $100 \mu \mathrm{m}$ optical depth is well correlated with the gas column density suggesting that the far-infrared emission must originate from a substantial depth in the clouds. The dust temperature is found to be anticorrelated with the gas column density indicating that these clouds are heated externally. Our calculated far-infrared luminosities of GF 17 and GF 20 imply that the dominant source of dust heating is the ISRF due to the nearby Sco OB2 association. Analysis of the gas velocity structure within GF 17 and GF 20 reveals evidence for smooth large-scale streaming motions along the filamentary structures with magnitude $\sim 0.5 \mathrm{~km} \mathrm{~s}^{-1} \mathrm{pc}^{-1}$. Our results indicate that the velocity gradients are likely due to the interaction of GF 17 and GF 20 with the Upper-Scorpius and Upper-Centaurus-Lupus HI expanding shells, via propagating shock fronts.
\end{abstract}

Key words. interstellar medium: clouds - interstellar medium: dust, extinction - interstellar medium: individual objects: Lupus clouds - interstellar medium: structure - interstellar medium: molecules

\section{Introduction}

Globular Filaments (GF) are nearby (100-700 pc distance) filamentary dark clouds containing small numbers of cloud cores connected by lower density gas and dust. Schneider \& Elmegreen (1979) compiled the first catalog of such clouds, containing 23 globular filaments. These clouds look like strings (the low opacity material) with beads (the denser cores) strung along their lengths, often in a periodic fashion. They may represent swept-up material created by blast waves or strong stellar winds (Schneider \& Elmegreen 1979; Heiles 1997) or they could be a natural stable state for magnetized galactic molecular clouds.

At an estimated distance of $150 \mathrm{pc}$, the Lupus complex of molecular dark clouds, which is located near the Scorpio-Centaurus (or Sco OB2) OB association (Humphreys 1978), is one of the most active nearby

\footnotetext{
Send offprint requests to: M. C. Moreira,

e-mail: miguelm@oal.ul.pt

* Based on observations at the European Southern Observatory, La Silla, Chile.
}

low-mass star-forming regions (Schwartz 1977; Krautter 1991; Hughes et al. 1994; Wichmann et al. 1997; Krautter et al. 1997). This complex consists of five filamentary dark clouds (Tachihara et al. 1996), and among these are globular filaments GF 17 and GF 20, also known as Lupus 4 and Lupus 2 dark clouds, respectively. GF 17 is a large filament, spanning about $1.5^{\circ}$ in the plane of the sky (Fig. 1), while GF 20 is a smaller filament extending for about $40^{\prime}$. The filamentary structure is particularly well delineated in GF 20 (Fig. 2) where a chain of small globules can be seen extending to the northeast and away from a larger condensation. Apparently there is no evidence for interaction with the surrounding environment in both GF 17 and GF 20 (Schneider \& Elmegreen 1979). A total of eight $\mathrm{T}$ Tauri stars are known to be associated with GF 17, and four young stellar objects are associated with GF 20.

Despite several studies dedicated to this star forming region, observations of molecular emission in Lupus have been rather limited, probably due to its southernly declination $\left(<-30^{\circ}\right)$. The Lupus complex of dark clouds was surveyed in the $\mathrm{CO}(1-0)$ line over an area of $\sim 170 \mathrm{sq} \mathrm{deg}$ by Murphy et al. (1986) with an effective resolution of 


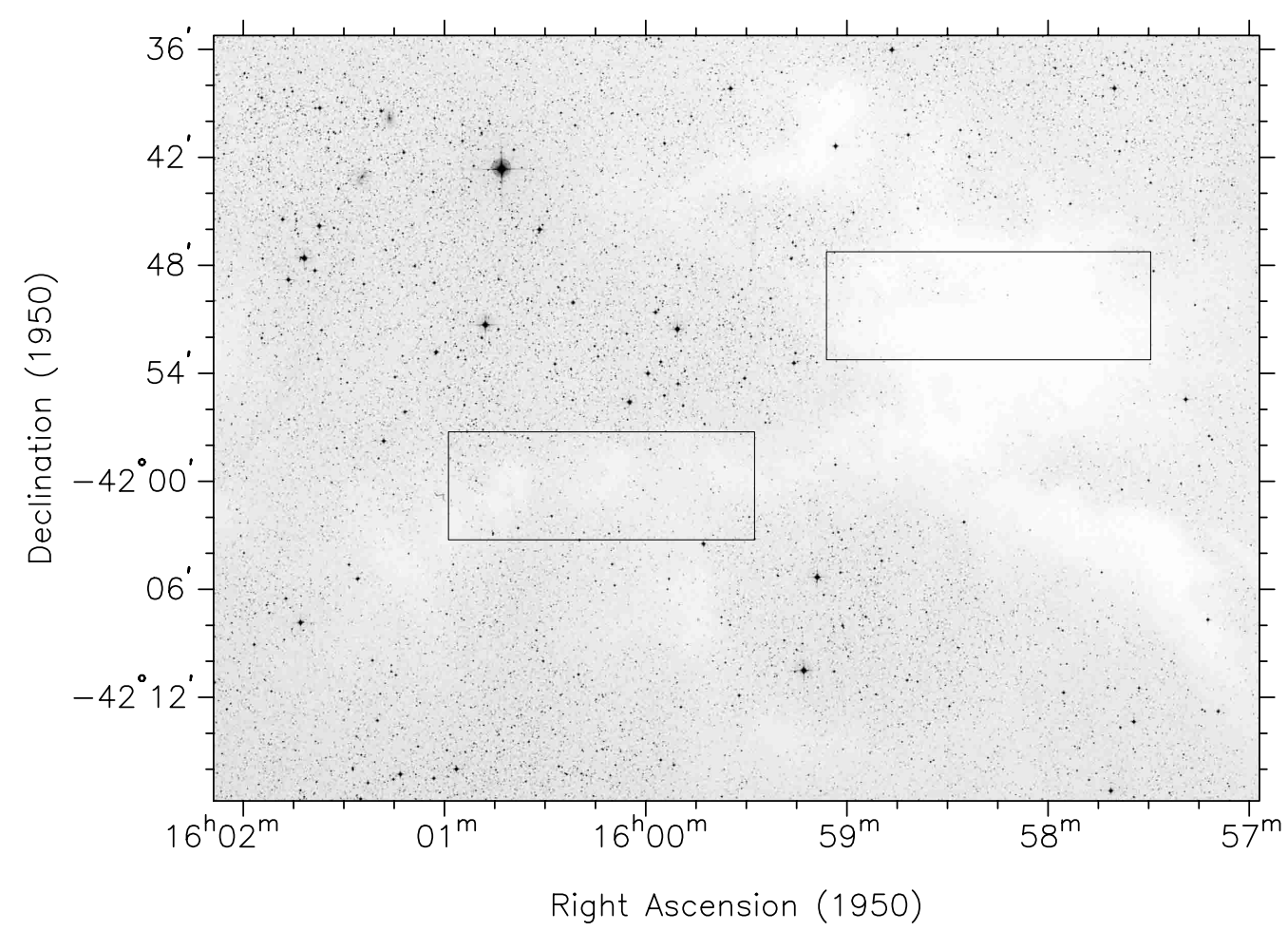

Fig. 1. Digital Sky Survey image of the central region of globular filament GF 17. The two rectangular boxes indicate the regions mapped in ${ }^{13} \mathrm{CO}$ in the present study. The region within the eastern box is referred to as the filamentary region, and the region within the western box as the main core region.

$30^{\prime}$. They estimated the mass of the cloud complex to be $3 \times 10^{4} M_{\odot}$, comparable to that of the nearby Ophiuchus complex of dark clouds. More recently, Tachihara et al. (1996) performed large-scale ${ }^{13} \mathrm{CO}(1-0)$ observations of the complex (with the exception of GF 17) with an effective resolution of $8^{\prime}$, and Gahm et al. (1993) made $\mathrm{CO}(1-0)$ observations of GF 20 with $40^{\prime \prime}$ resolution but covering only the main condensation of the cloud. Using optical star counts, Andreazza \& Vilas-Boas (1996) derived masses of $197 M_{\odot}$ and $59 M_{\odot}$ for GF 17 and GF 20, respectively.

The main purpose of these few sudies was to better characterize the star formation activity within the dark clouds in Lupus. However, the Lupus 1 and 3 dark clouds have been much more actively engaged in star formation (see Krautter 1991, for a review) than GF 17 or GF 20. Consequently, the physical properties (such as gas kinematics and temperatures, and density structure) of the molecular material as well as the dust content of the GF 17 and GF 20 globular filaments remain virtually unknown.

As part of a comprehensive study of the structure, physical conditions, and dynamical states of dark globular filaments (Moreira et al. 2000; Moreira \& Yun 2002), we have performed large-scale observations of the physical properties of the gas and dust within GF 17 and GF 20. The elements of our study were (1) 12, 25, 60, and $100 \mu \mathrm{m}$ IRAS co-added images, and (2) large-scale millimeter molecular line maps in the $J=1-0$ transition of $\mathrm{CO},{ }^{13} \mathrm{CO}$, and $\mathrm{C}^{18} \mathrm{O}$ with unprecedented spatial coverage and resolution. In this paper we present and discuss the correlations between gas and dust in GF 17 and GF 20. Analysis of the gas velocity structure in GF 17 and GF 20 strongly indicates that these clouds have been shaped by the interaction with expanding HI shells originated in the nearby Sco OB2 association.

In Sect. 2 we present the observations and data reduction. Our method of analysis of the IRAS co-added images and millimeter molecular line data is presented in Sect. 3. In Sects. 4 and 5 we present and discuss our results. Section 6 summarizes our findings.

\section{Observations and data reduction}

\subsection{IRAS data acquisition and image processing}

IRAS co-added images at 12, 25, 60 and $100 \mu \mathrm{m}$ were obtained from the Infrared Processing and Analysis Center $(\mathrm{IPAC})^{1}$. The images were interpolated to a pixel size of $15^{\prime \prime} \times 15^{\prime \prime}$. Our image processing procedure, which is similar to the procedures developed by Langer et al. (1989), Snell et al. (1989), and Wood et al. (1994) has two steps. First, we remove the emission from zodiacal dust in the 60 and $100 \mu \mathrm{m}$ IRAS images of each field. Next, we subtract a background from all images in order to ensure that empty sky has zero surface brightness.

The images in our study exhibit only marginal smooth large-scale brightness gradients produced by zodiacal dust. The $60 \mu \mathrm{m}$ images are generally more affected by

${ }^{1}$ IPAC is funded by NASA as part of the IRAS extended mission program under contract to JPL. 


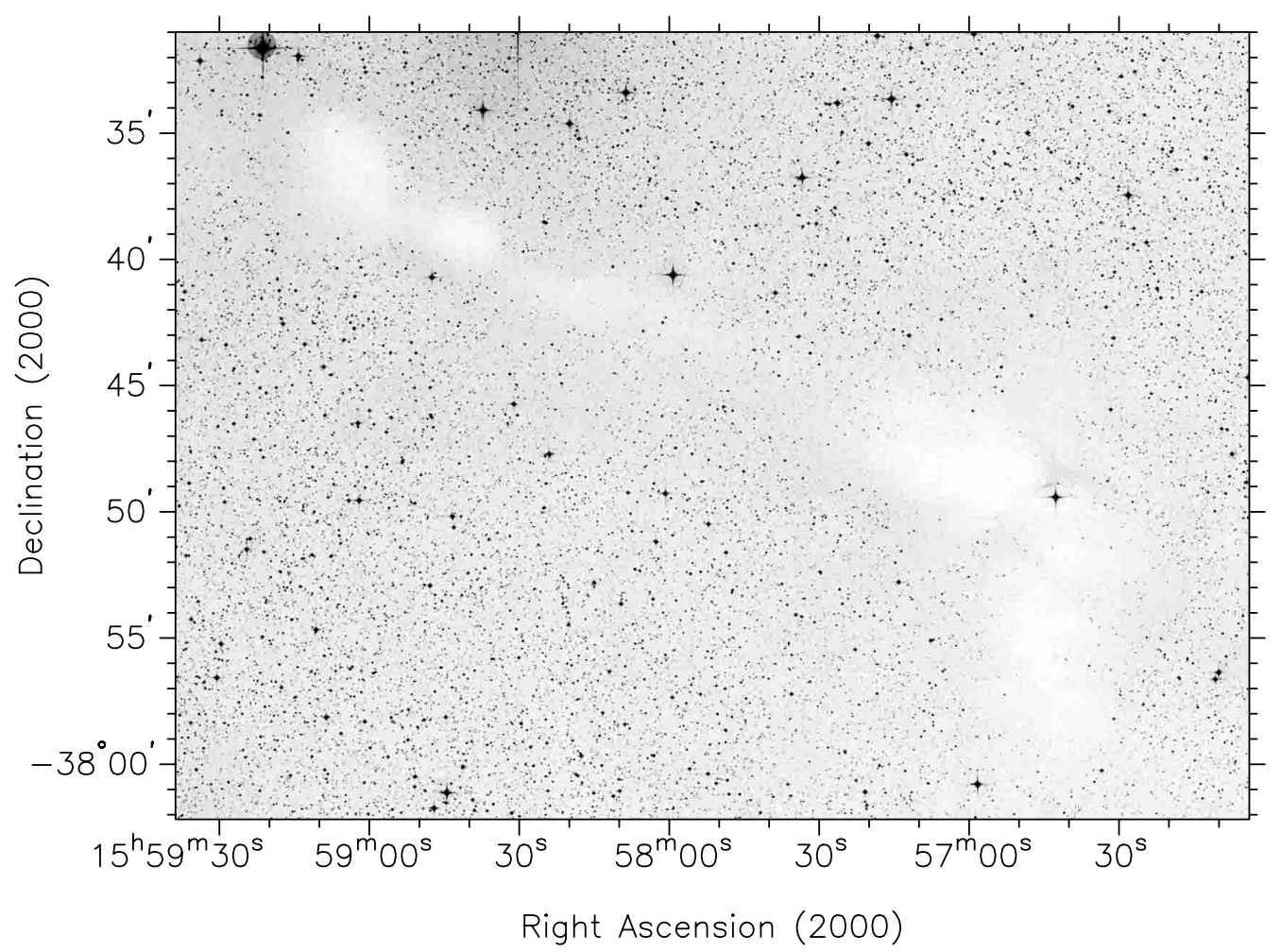

Fig. 2. Digital Sky Survey image of the globular filament GF 20.

zodiacal dust than the $100 \mu \mathrm{m}$ images. Before we calculate dust temperature and opacity images, the contribution due to zodiacal and other background emission unrelated to GF 17 and GF 20 should be removed. Fortunately, the temperature of zodiacal dust, $\sim 150 \mathrm{~K}$, is very different from the $\sim 30 \mathrm{~K}$ dust found in dark clouds, and unlike most dark clouds, zodiacal dust emission is generally smooth and very large-scale (i.e., structures that span several degrees). However, since GF 17 and GF 20 lie more than $20^{\circ}$ away from the plane of the ecliptic, the zodiacal light contribution to our images is minimal and can safely be ignored. To test this assumption, we selected 3 points in each $100 \mu \mathrm{m}$ image that appeared to have no dust emission. The mean value of the image in a $10^{\prime} \times 10^{\prime}$ box at each of the 3 positions was determined. We then calculated the image of a plane that passes through these points and subtracted this "flat-field" from the $100 \mu \mathrm{m}$ image. For the $60 \mu \mathrm{m}$ images we calculated the plane at the same 3 positions used for the $100 \mu \mathrm{m}$ image, and subtracted this "flat-field" from the corresponding $60 \mu \mathrm{m}$ image. We find that the calculated dust temperatures of the clouds vary by less than $2 \mathrm{~K}$ (or $5 \%$ ). Thus, the errors are negligible and zodical light can be ignored.

The zero level of the IRAS images is somewhat arbitrary. In order to obtain images in which apparently empty sky has a surface brightness of zero, we offset the background level in each field. Using the $100 \mu \mathrm{m}$ image as a reference (because it shows cold cloud structures better than the $60 \mu \mathrm{m}$ image), we calculated the minimum flux values in the $12,25,60$ and $100 \mu \mathrm{m}$ images. The minimum flux value was then subtracted from each image to produce the final 12, 25, 60 and $100 \mu \mathrm{m}$ images. To insure that the images were treated the same, we used the same positions in all the images of a field when evaluating the background level.

\subsection{Millimeter data}

Observations of GF 17 and GF 20 were carried out in four different periods, during 1996 January 20-25 and July 10-13, 1997 January 25-28, and 1999 January 19-24 at La Silla, ESO, Chile. Observations of the CO $(J=1-0)$, ${ }^{13} \mathrm{CO}(J=1-0)$, and $\mathrm{C}^{18} \mathrm{O}(J=1-0)$ rotational transitions (hereafter, $\mathrm{CO},{ }^{13} \mathrm{CO}$, and $\mathrm{C}^{18} \mathrm{O}$ ) were performed with the $15 \mathrm{~m}$ SEST telescope. The SEST and its instrumentation have been described in detail by Booth et al. (1989) and an update was given by Nyman \& Booth (1990). A high-resolution 2000-channel acoustooptical spectrometer (AOS) was used as a back end, with a total bandwidth of $86 \mathrm{MHz}$ and a resolution of $43 \mathrm{kHz}$ per channel. SIS receivers were used in single side-band (SSB) mode. Typical SSB system temperatures were found to be in the range $160-400 \mathrm{~K}$ during the observations. The antenna half-power beamwidth is $44^{\prime \prime}$ for $\mathrm{CO}$ and $46^{\prime \prime}$ for ${ }^{13} \mathrm{CO}$ and $\mathrm{C}^{18} \mathrm{O}$. Typical signal-to-noise ratios were 60,50 , and 20 for $\mathrm{CO},{ }^{13} \mathrm{CO}$, and $\mathrm{C}^{18} \mathrm{O}$, respectively. The main beam efficiency $\left(\eta_{\mathrm{mb}}\right)$ was 0.7 at the frequencies of the $J=1-0$ transition of $\mathrm{CO},{ }^{13} \mathrm{CO}$, and $\mathrm{C}^{18} \mathrm{O}$.

We have obtained CO spectra toward 316 and 522 positions in GF 17 and GF 20, respectively. For ${ }^{13} \mathrm{CO}$, a total of 356 spectra were taken in GF 17, and 391 spectra were obtained for GF $20 . \mathrm{C}^{18} \mathrm{O}$ emission was searched in 
139 positions in GF 17 and in 274 positions toward GF 20. The clouds were mapped with $46^{\prime \prime}$ spacing, corresponding approximately to full-beam spacing at the frequencies of the $\mathrm{CO},{ }^{13} \mathrm{CO}$, and $\mathrm{C}^{18} \mathrm{O}$ line transitions. In GF 17 , two regions (delimited by the boxes in Fig. 1) were searched for CO emission, while GF 20 was virtually completely mapped. The spectra were taken in frequency-switching mode (with frequency throws in the range $10-16 \mathrm{MHz}$ ). The spectral line intensities were calibrated and corrected for atmospheric losses using the standard chopper wheel method (Kutner \& Ülich 1981). The observed line intensities are expressed as antenna temperature $T_{\mathrm{A}}^{*}$. Division of $T_{\mathrm{A}}^{*}$ by $\eta_{\mathrm{mb}}$ yields the main beam radiation temperature, $T_{\mathrm{mb}}$. Pointing was checked every $2-3$ hours by means of repeated spectral line observations of the $\mathrm{SiO}$ $(v=1, J=2-1)$ maser sources W Hya, R Dor, L2 Pup, and Vx Sgr, and was found to be accurate to within $6^{\prime \prime}$. The data were processed using standard procedures of the Continuum and Line Analysis Single-dish Software (CLASS) package developed at Observatoire de Grenoble and IRAM Institute. The spectra were folded, and baselines of order $\leq 3$ were fitted and removed. Upon fitting a baseline, each observed spectral line was fitted by a gaussian profile using a non-linear least chi-square $\left(\chi^{2}\right)$ minimization, yielding the antenna temperature, central velocity, and linewidth of the line.

\section{Derived parameters of the clouds}

\subsection{Dust temperature}

The 60 and $100 \mu \mathrm{m}$ dust color temperature, $T_{\mathrm{d}}$, was calculated at each pixel in an image assuming that the dust in a single beam can be characterized by one single temperature $\left(T_{\mathrm{d}}\right)$, and that the emission at 60 and $100 \mu \mathrm{m}$ is due to blackbody radiation from dust grains at temperature $T_{\mathrm{d}}$, modified by a power-law emissivity. The flux density of optically thin emission from dust grains at wavelength $\lambda$ is given by (e.g. Arce \& Goodman 1999)

$F_{\lambda}=B_{\lambda}\left(T_{\mathrm{d}}\right) N_{\mathrm{d}} \tau_{\lambda} \Omega_{\lambda} \propto B_{\lambda}\left(T_{\mathrm{d}}\right) N_{\mathrm{d}} \lambda^{-\beta} \Omega_{\lambda}$,

where $N_{\mathrm{d}}$ is the column density of dust grains, $B_{\lambda}\left(T_{\mathrm{d}}\right)$ is the Planck function, $\tau_{\lambda}$ is the dust optical depth, $\Omega_{\lambda}$ is the solid angle at $\lambda$, and $\beta$ is the power-law index of the dust emissivity. If we calculate the ratio, $R$, of the flux densities at $100 \mu \mathrm{m}$ and $60 \mu \mathrm{m}$ (after smoothing the $60 \mu \mathrm{m}$ images to the spatial resolution of the $100 \mu \mathrm{m}$ images), we can construct a look-up table with the value of $R$ calculated for a wide range of $T_{\mathrm{d}}$. For each pixel in the image, the table is then searched for the value of $T_{\mathrm{d}}$ that reproduces the observed 60 to $100 \mu \mathrm{m}$ flux ratio at each pixel. Emissivities $\epsilon \propto \lambda^{-1}$ and $\epsilon \propto \lambda^{-2}$ were used to calculate two dust temperatures for each globular filament.

\subsection{Dust optical depth and visual extinction}

Assuming optically thin emission, we use the dust color temperature to calculate the dust optical depth at each pixel

$\tau_{100}=10^{-17} \frac{F_{\lambda}(100 \mu \mathrm{m})}{B_{\lambda}\left(100 \mu \mathrm{m}, T_{\mathrm{d}}\right)}$,

where $B_{\lambda}\left(100 \mu \mathrm{m}, T_{\mathrm{d}}\right)$ is the Planck function and $F_{\lambda}(100 \mu \mathrm{m})$ is the observed $100 \mu \mathrm{m}$ flux. We then use Eq. (5) of Wood et al. (1994) to convert from dust optical depth to visual extinction:

$A_{V}=15.078\left(1-\mathrm{e}^{-\tau_{100} / 641.3}\right)$,

where $\tau_{100}$ is the optical depth given in units of $10^{-6}$. This result is obtained by fitting the relation between $60 \mu \mathrm{m}$ optical depth $\left(\tau_{60}\right)$ and $A_{V}$ found by Jarrett et al. (1989) for the $\rho$ Ophiuchi region. Assuming optically thin emission, Wood et al. (1994) multiply the Jarrett et al. (1989) $\tau_{60}$ values by $100 / 60$ to convert to $\tau_{100}$ and obtain Eq. (3) above. Thus, the derived extinction values are subject to the uncertainties in the conversion equation. However, Jarrett et al. (1989) show that there is a very tight correlation between $\tau_{60}$ and $A_{V}$ for $A_{V} \leq 5 \mathrm{mag}$, implying very little uncertainty in the conversion of farinfrared optical depth to visual extinction, at least in the low $\left(A_{V} \leq 5 \mathrm{mag}\right)$ extinction regions of GF 17 and GF 20 .

\subsection{Errors and assumptions}

In order to derive the dust temperature and optical depths, we have made the following assumptions: (1) the dust is optically thin at 60 and $100 \mu \mathrm{m},(2)$ dust emissivity is proportional to a power law $\lambda^{-\beta}$, with index $\beta=1$, and (3) the dust in the IRAS beam is at a single temperature. We discuss the validity of these assumptions below.

Draine \& Lee (1984) have shown that the $100 \mu \mathrm{m}$ optical depth can be estimated in terms of the hydrogen column density along the line of sight as $\tau_{100}=$ $3.6 \times 10^{-25} N_{\mathrm{H}}$. Thus, $\tau_{100} \sim 1$ only for $N_{\mathrm{H}}$ in excess of $2.7 \times 10^{24} \mathrm{~cm}^{-2}$, which is well above typical Galactic plane values. In addition, the largest $\tau_{100}$ we find in our images is $\sim 3 \times 10^{-5}$. Thus, assumption (1) is valid.

The errors introduced by assuming a constant $\beta$ along the line of sight are hard to estimate, since we do not have any way to measure how much $\beta$ changes in our regions of study. There is a general agreement that the emissivity index depends on the grain size, composition, and physical structure (Weintraub et al. 1991), and the general consensus in recent years has been that $\beta$ has a value most likely between 1 and 2 , that in the general ISM $\beta$ is close to 2 , and in denser regions with bigger grains $\beta$ is closer to 1 (Beckwith \& Sargent 1991; Mannings \& Emerson 1994; Pollack et al. 1994). We have performed tests with $\beta=2$ and find that our results are not significantly affected: for both clouds, the dust color temperature calculations with $\beta=2$ and $\beta=1$ are consistent within $20 \%$. Thus, we conclude that assumption (2) is acceptable. 
Assumption (3) is certainly not valid near local heat sources. Langer et al. (1989) and Draine (1990) have considered this problem by examining a simple twocomponent model where the two regions have different dust temperatures and optical depths. They have shown that the calculated dust temperature is dominated by the emission from the hot dust component, even if this component represents only a few percent of the total mass of dust. Essentially our temperature determination, from which we calculate the $100 \mu \mathrm{m}$ and $60 \mu \mathrm{m}$ emission, is an emissivity weighted rather than a mass weighted dust temperature. Thus, our single-temperature assumption is violated in the immediate vicinity of stars embedded in the cloud. If a star heats the dust in its vicinity, the calculated $T_{60 / 100}$ will be dominated by the hot dust, the derived $\tau_{100}$ will be too low and consequently $A_{V}$ will be underestimated. Hence, the masses of the clouds that have embedded stars with unresolved dust temperature gradients will also be underestimated. Even away from the point sources, complex temperature structure may be present and must be kept under consideration when interpreting the derived dust optical depths and temperatures. Without higher spatial resolution observations or modeling the dust temperature distribution close to embedded stars we cannot remove this effect.

\subsection{Gas column densities}

In estimating the physical parameters of the molecular gas in GF 17 and GF 20, we have assumed that the main beam temperature, $T_{\mathrm{mb}}$, is a good approximation of the source brightness temperature, since the molecular structures observed are extended with respect to the telescope beam. We assume a plane-parallel, LTE, isothermal, and optically thin radiative transfer model for the ${ }^{13} \mathrm{CO}$ transition line.

The excitation temperature of each line of sight in each cloud was computed from the peak CO intensity under the assumptions of high optical depth of the $\mathrm{CO}$ line and a beam-filling factor of unity, using the equation

$\frac{T_{0}}{T_{\mathrm{ex}}}=\ln \left[1+\frac{T_{0}}{J\left(T_{\mathrm{bg}}\right)+T_{\mathrm{mb}}}\right]$

where $T_{0}=h \nu / k, T_{\mathrm{bg}}$ is the background temperature, which we take to be $2.73 \mathrm{~K}$, and $J(T)$ is given by $J(T)=$ $(h \nu / k)[\exp (h \nu / k T)-1]^{-1}$. For densities typical of these dark clouds, at least in the dense cores, the CO is expected to be thermalized by collisions at the gas kinetic temperature, so that the excitation temperature should be close to the kinetic temperature of the gas, $T_{\mathrm{ex}} \simeq T_{\mathrm{k}}$. By averaging all positions in each cloud, we find mean excitation temperatures of $16.8 \mathrm{~K}$ and $17.7 \mathrm{~K}$ for GF 17 and GF 20 , respectively.

From the ${ }^{13} \mathrm{CO}$ observations, we can obtain the column densities of the cloud with the usual assumptions that (1) CO is optically thick, $(2){ }^{13} \mathrm{CO}$ is optically thin, (3) the $\mathrm{CO}$ and ${ }^{13} \mathrm{CO}$ transitions have the same excitation temperatures, and (4) the same beam filling factor.
The optical depth of the ${ }^{13} \mathrm{CO}$ line can be obtained by using the measured temperatures of $\mathrm{CO}$ and ${ }^{13} \mathrm{CO}$ at each position toward the cloud:

$$
\frac{T_{\mathrm{mb}}(\mathrm{CO})}{T_{\mathrm{mb}}\left({ }^{13} \mathrm{CO}\right)} \approx \frac{\left[1-\exp \left(-X \tau_{13}\right)\right]}{\left[1-\exp \left(-\tau_{13}\right)\right]}
$$

where $\tau_{13}$ is the optical depth of the ${ }^{13} \mathrm{CO}$ transition, and $X$ is the isotopic abundance ratio of the two species, which we assume to be about 89 (Gahm et al. 1993). We find that CO is optically very thick across the entire mapped regions, with $\tau_{12} \sim 90$ near the densest regions of GF 17 and GF 20.

Combining $\tau_{13}$ and $T_{\mathrm{ex}}$, the LTE column density of ${ }^{13} \mathrm{CO}$ can be estimated as (e.g. Bourke et al. 1997)

$N\left({ }^{13} \mathrm{CO}\right)=2.42 \times 10^{14} \frac{T_{\mathrm{ex}}+0.88}{1-\exp \left(-5.29 / T_{\mathrm{ex}}\right)} \int \tau_{13} \mathrm{~d} v$,

where $v$ is measured in $\mathrm{km} \mathrm{s}^{-1}$. From this, we obtain $\mathrm{H}_{2}$ column densities in GF 17 and GF 20 by assuming an $\left[\mathrm{H}_{2} /{ }^{13} \mathrm{CO}\right]$ ratio of $7 \times 10^{5}$ (Frerking et al. 1982).

We derive an estimate of the cloud mass from the ${ }^{13} \mathrm{CO}$ observations by integrating Eq. (6) over the solid angle subtended by the source. For the areas covered by our ${ }^{13} \mathrm{CO}$ maps, we find gas masses of $36 M_{\odot}$ and $31 M_{\odot}$ for GF 17 and GF 20, respectively, assuming a distance of $150 \mathrm{pc}$ to the Lupus complex. In GF 17 , our ${ }^{13} \mathrm{CO}$ maps are limited to a region which is much smaller than the full extent of the cloud. Assuming similar conditions outside the areas covered in this study, the total mass of GF 17 may amount to $\sim 200 M_{\odot}$, in good agreement with the estimate by Andreazza \& Vilas-Boas (1996), and we regard this as an upper limit since the density is seen to drop significantly towards the cloud boundaries. On the other hand, GF 20 (which was almost completely mapped) has very low mass, a factor of $\sim 3$ smaller than the previous estimate by Tachihara et al. (1996).

\section{Results and analysis}

Figures 3 and 4 present the 12, 25, 60, and $100 \mu \mathrm{m}$ background-subtracted IRAS co-added images of GF 17 and GF 20. The $12 \mu \mathrm{m}$ and $25 \mu \mathrm{m}$ images reveal few point sources toward GF 17 and GF 20. Some of these sources are confirmed young stellar objects (mostly T Tauri stars) associated with their parent cloud, while other sources are simply unrelated background and/or foreground objects. The locations of confirmed young stellar objects within each cloud are indicated by the filled star symbols. Of the eight $\mathrm{T}$ Tauri stars known to be associated with GF 17 (Schwartz 1977), only four are seen in our IRAS field. These are found in regions of visual extinction smaller than 1 mag (Andreazza \& Vilas-Boas 1996), and distributed around the main core region of GF 17. Four of the five T Tauri stars associated with GF 20 are seen in our IRAS field. The strongest $25 \mu \mathrm{m}$ source, and almost coincident with the peak of $100 \mu \mathrm{m}$ emission, is the extremely active T Tauri star RU Lupi (Schwartz 1977). 
$12 \mu \mathrm{m}$

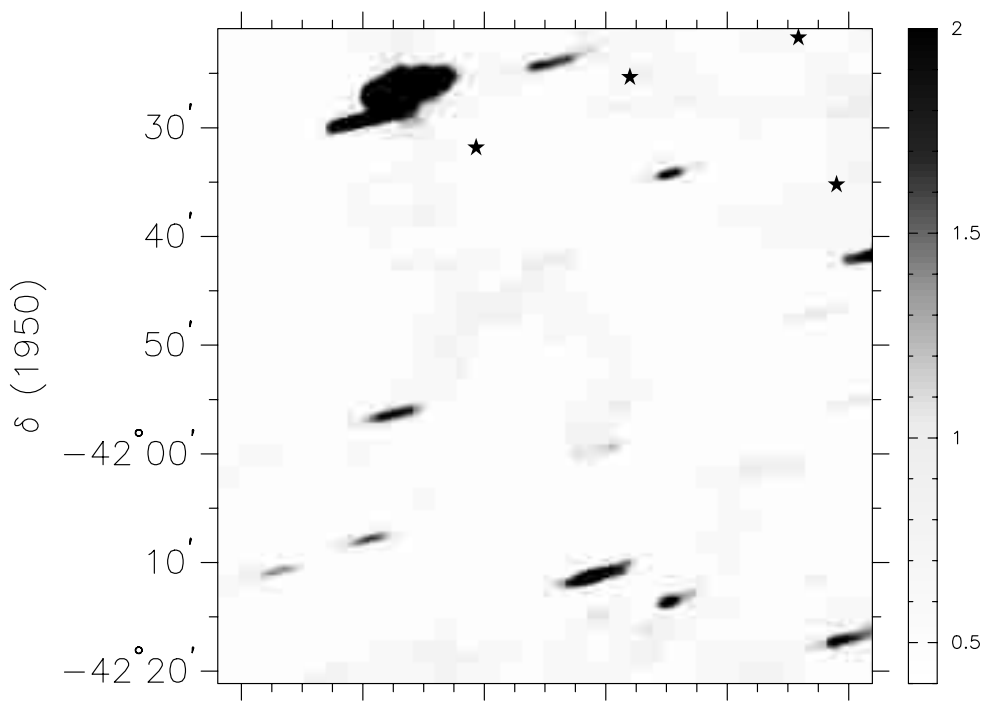

$25 \mu \mathrm{m}$

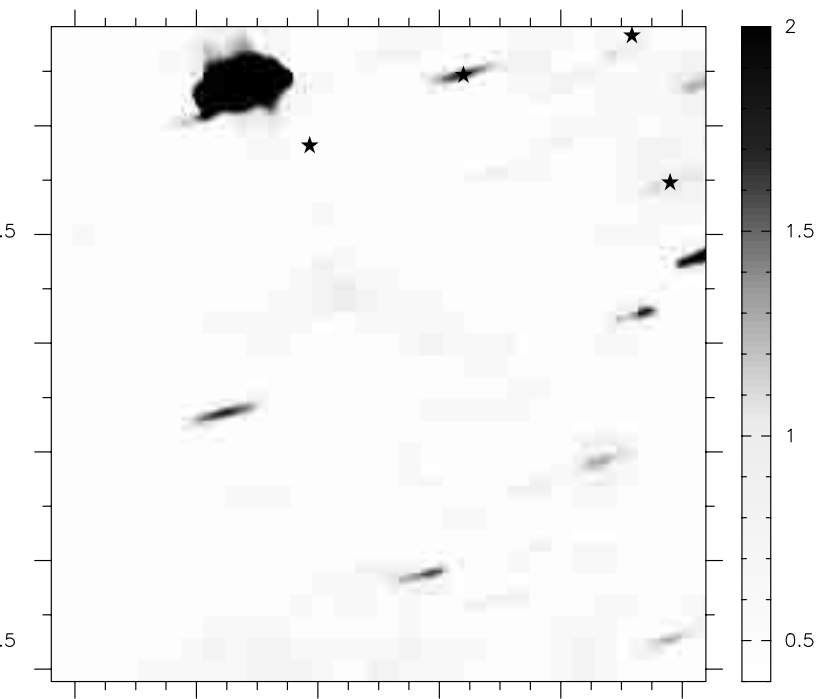

$60 \mu \mathrm{m}$

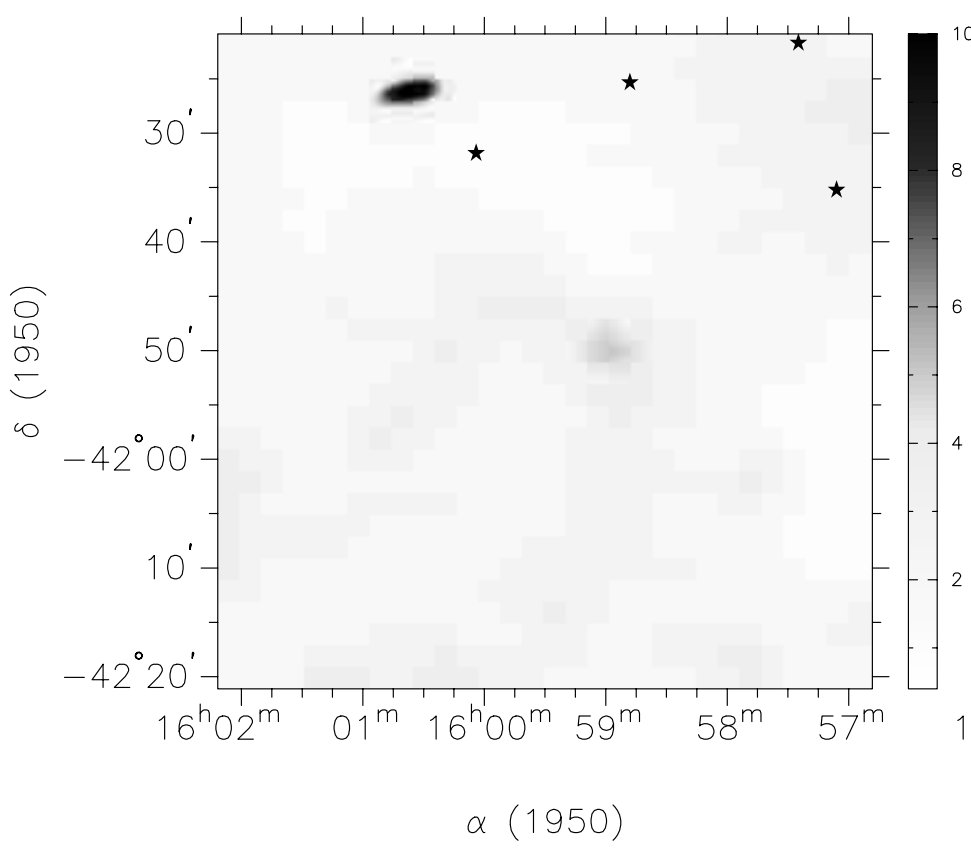

$100 \mu \mathrm{m}$

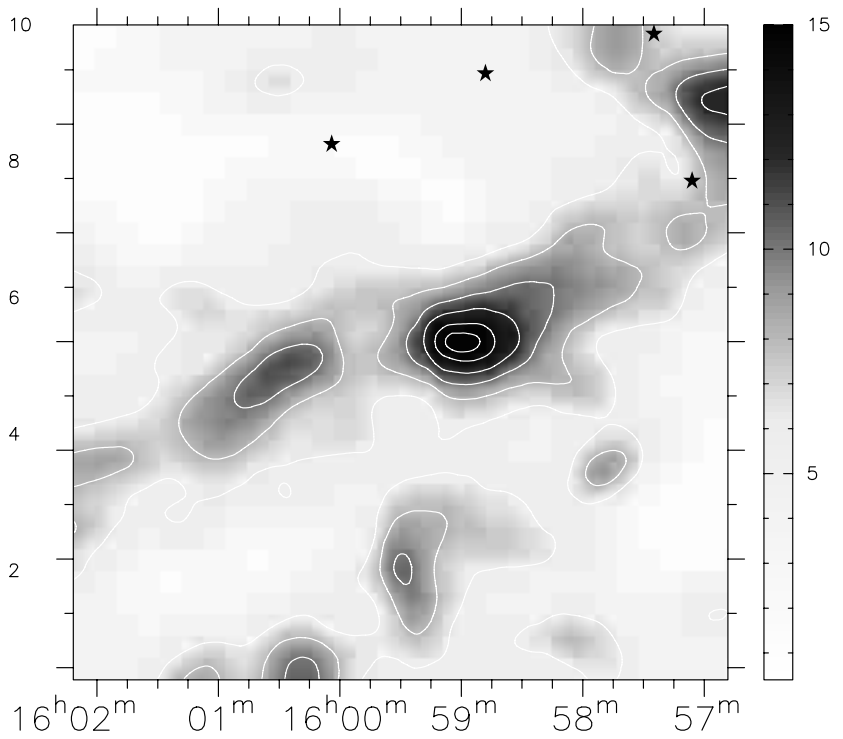

$\alpha(1950)$

Fig. 3. Background-adjusted IRAS co-added images obtained toward GF 17. Filled stars show the positions of T Tauri stars associated with GF 17 . The intensity scale in each panel is $10^{6} \mathrm{Jy} \mathrm{ster}^{-1}$, starting at $0.4 \times 10^{6} \mathrm{Jy}_{\text {ster }}{ }^{-1}$ (mean noise level). For clarity, contours of $100 \mu \mathrm{m}$ emission at $6,8,10,12,14$, and $15 \times 10^{6} \mathrm{Jy} \mathrm{ster}^{-1}$ are shown.

Andreazza \& Vilas-Boas (1996) estimate the visual extinction to this source to be $\sim 3.8$ mag from optical star counts. The remaining $\mathrm{T}$ Tauri stars are found in regions of visual extinction smaller than 1 mag (Andreazza \& Vilas-Boas 1996).

\subsection{IRAS cloud morphology}

The IRAS wide-field, high dynamic range images clearly reveal the filamentary nature of GF 17 and GF 20. The spatial distribution of dust emission in these globular filaments is not irregular in shape, but rather is extremely elongated. The $100 \mu \mathrm{m}$ emission from GF 17 peaks at a main core region, and defines a filamentary region toward the east. In GF 20, the $100 \mu \mathrm{m}$ emision peaks at a main core region associated with RU Lupi. A second strong peak of $100 \mu \mathrm{m}$ emission is located about $5^{\prime}$ to the south of RU Lupi. A third peak of $100 \mu \mathrm{m}$ emission appears within the filamentary part of GF 20 . Like GF 17 , the filamentary structure in GF 20 is well delineated at $100 \mu \mathrm{m}$, extending to the northeast, away from RU Lupi. Table 1 summarizes the $60 \mu \mathrm{m}$ and $100 \mu \mathrm{m}$ peak fluxes of dust emission from 
$12 \mu \mathrm{m}$

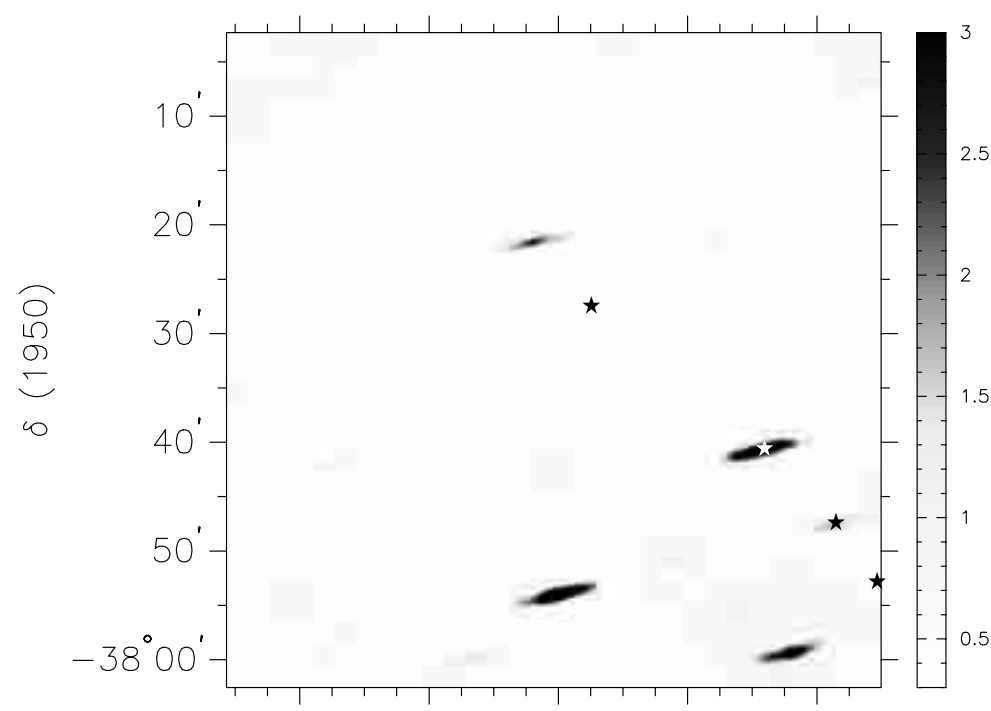

$60 \mu \mathrm{m}$

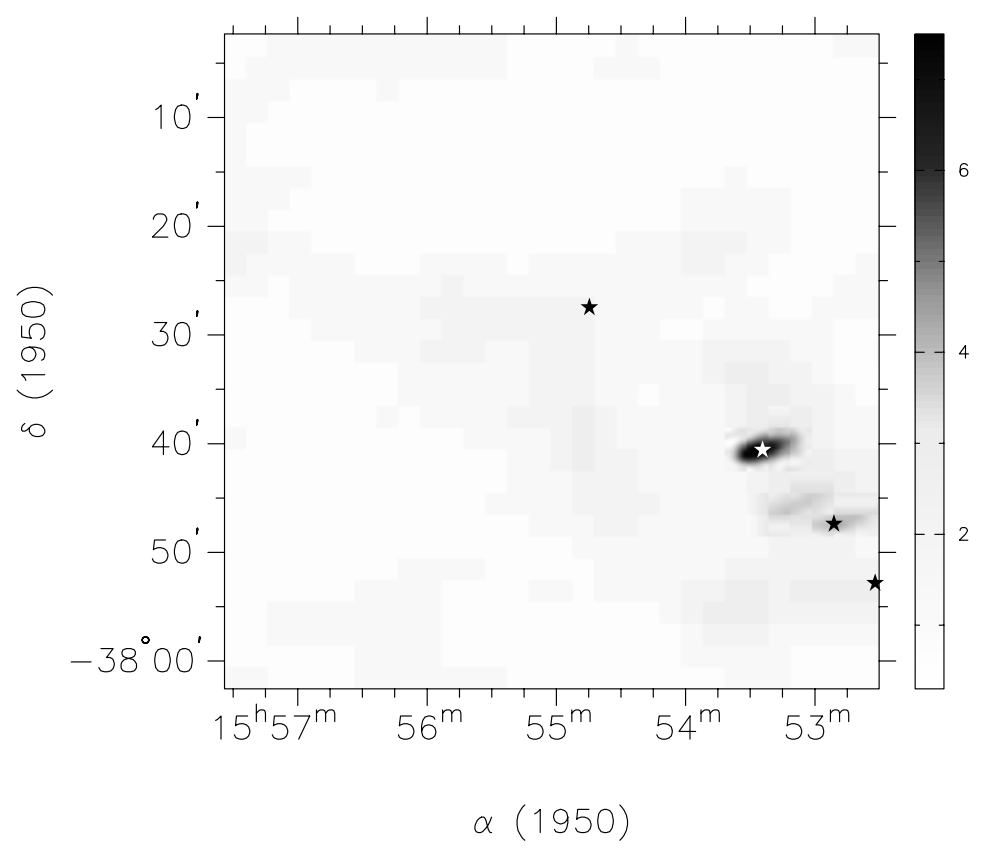

$25 \mu \mathrm{m}$

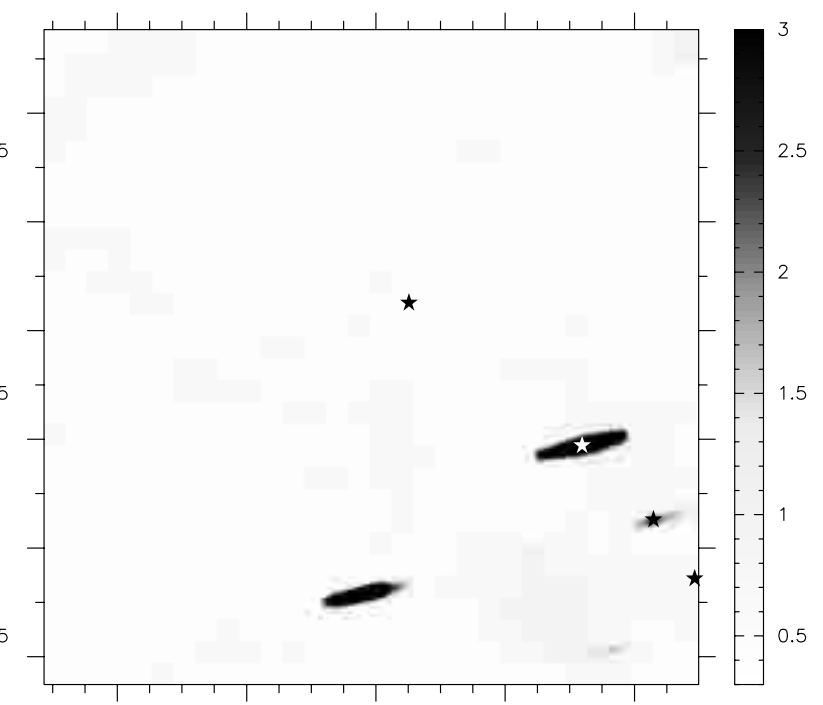

$100 \mu \mathrm{m}$

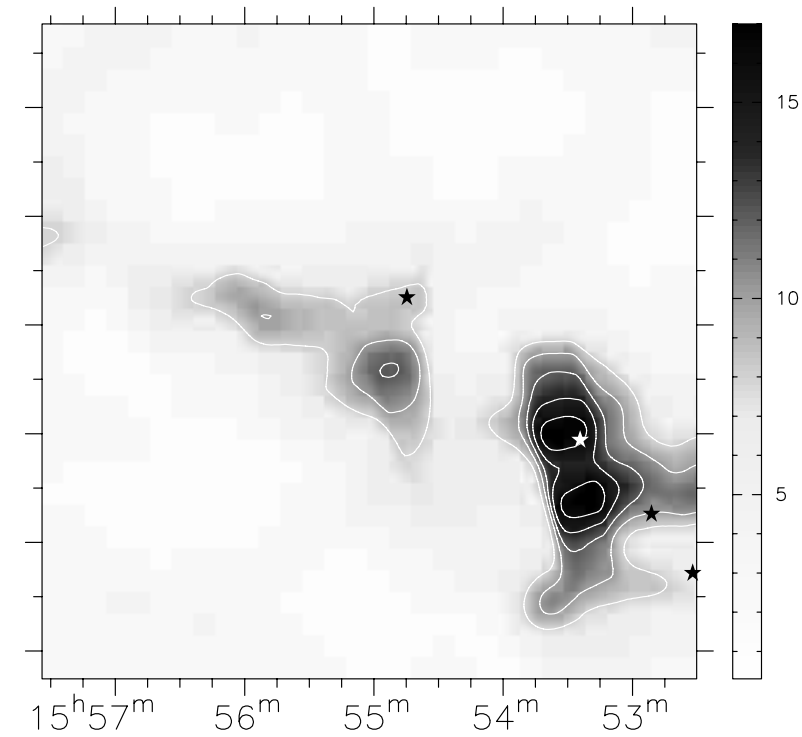

$\alpha(1950)$

Fig. 4. Background-adjusted IRAS co-added images obtained toward GF 20. Filled stars show the positions of T Tauri stars associated with GF 20. The white star indicates the position of the extremely active T Tauri star RU Lupi. The intensity scale in each panel is $10^{6} \mathrm{Jy} \mathrm{ster}^{-1}$, starting at $0.3 \times 10^{6} \mathrm{Jy} \mathrm{ster}^{-1}$ (mean noise level). For clarity, contours of $100 \mu \mathrm{m}$ emission at 8 , $10,12,14$, and $16 \times 10^{6}$ Jy ster $^{-1}$ are shown.

selected regions within each cloud. The positions in Cols. 3 and 4 were derived from the $100 \mu \mathrm{m}$ images. Columns 7, 8, and 9 give average dust temperatures, optical depths, and visual extinctions observed within each region.

GF 17 and GF 20 share an interesting characteristic in their dust emission. Note that we do not see the clouds boundaries at 12 or $25 \mu \mathrm{m}$. This is unlike the $\rho$ Ophiuchi cloud which has IR boundaries clearly delineated at both $12 \mu \mathrm{m}(h c / k \lambda \sim 1000 \mathrm{~K})$ and $100 \mu \mathrm{m}(h c / k \lambda \sim 100 \mathrm{~K})$, as shown by Jarrett et al. (1989). These authors concluded that the IR emission from $\rho$ Ophiuchi needs to be modeled as arising from two physically distinct populations of dust grains. Our IRAS images thus suggest that the IR emission from GF 17 and GF 20 can be modeled as arising from one single population of "cool" dust grains, and we proceed with the assumption that the emission at $60 \mu \mathrm{m}$ and $100 \mu \mathrm{m}$ probably arises from large dust grains in equilibrium with the radiation field. However, 
Table 1. Far-infrared properties of GF 17 and GF 20.

\begin{tabular}{|c|c|c|c|c|c|c|c|c|}
\hline \multirow[b]{2}{*}{$\begin{array}{l}\text { Cloud } \\
(1)\end{array}$} & \multirow[b]{2}{*}{$\begin{array}{c}\text { Region } \\
(2)\end{array}$} & \multirow{2}{*}{$\begin{array}{c}\text { RA } \\
(1950) \\
(3) \\
\end{array}$} & \multirow{2}{*}{$\begin{array}{c}\text { Dec } \\
(1950) \\
(4) \\
\end{array}$} & \multicolumn{2}{|c|}{$\begin{array}{c}\text { Peak Brightness } \\
\left.\text { (MJy ster }^{-1}\right)\end{array}$} & \multirow{2}{*}{$\begin{array}{c}T_{60 / 100} \\
(\mathrm{~K}) \\
(7) \\
\end{array}$} & \multirow{2}{*}{$\begin{array}{c}\tau_{100} \\
\left(\times 10^{-5}\right) \\
(8) \\
\end{array}$} & \multirow{2}{*}{$\begin{array}{c}A_{V} \\
(\mathrm{mag}) \\
(9) \\
\end{array}$} \\
\hline & & & & $\begin{array}{c}60 \mu \mathrm{m} \\
\quad(5)\end{array}$ & $\begin{array}{c}100 \mu \mathrm{m} \\
(6)\end{array}$ & & & \\
\hline \multirow[t]{2}{*}{ GF 17} & main core & $15^{\mathrm{h}} 59^{\mathrm{m}} 00^{\mathrm{s}}$ & $-41^{\circ} 50^{\prime}$ & 4.8 & 15.4 & 43 & 0.7 & 0.4 \\
\hline & filament & 160030 & $-41^{\circ} 52^{\prime}$ & 3.2 & 11.0 & 42 & 0.4 & 0.2 \\
\hline \multirow[t]{4}{*}{ GF 20} & main core ${ }^{(a)}$ & 155330 & $-37^{\circ} 47^{\prime}$ & 3.4 & 17.1 & 31 & 3.1 & 0.7 \\
\hline & main core ${ }^{(b)}$ & 155340 & $-37^{\circ} 38^{\prime}$ & 4.4 & 17.2 & 30 & 2.8 & 0.6 \\
\hline & filament & 155500 & $-37^{\circ} 33^{\prime}$ & 2.3 & 12.1 & 33 & 1.6 & 0.4 \\
\hline & filament & 155600 & $-37^{\circ} 28^{\prime}$ & 1.8 & 10.0 & 32 & 1.5 & 0.4 \\
\hline
\end{tabular}

(a) South of RU Lupi.

(b) Associated with RU Lupi.

we caution that part of the $60 \mu \mathrm{m}$ emission may arise from transiently excited particles (Puget \& Léger 1989).

\subsection{Dust color temperature and optical depth}

As mentioned above, the derived temperatures should be viewed with a great deal of caution. For the optically thin emission detected from these clouds by IRAS, the exponential nature of the flux dependence on temperature leads to a bias toward higher derived temperatures than are physically present along the line of sight. Hence, all the temperatures derived are weighted toward the warmer parts of the clouds and not the mass-averaged bulks of the clouds. Note, then, that all temperatures are upper limits, and all opacities are lower limits.

Figures 5 and 6 present images of the dust color temperature, $T_{60 / 100}$, and dust optical depth, $\tau_{100}$, for GF 17 and GF 20, respectively. The dust temperatures we derive are in reasonable agreement with the range of temperatures $(20-40 \mathrm{~K})$ derived by Jarrett et al. (1989) for the $\rho$ Oph cloud, and larger than the values $(20-25 \mathrm{~K}$ ) found by Wood et al. (1994) for the L1521 and L1506 filaments in Taurus. Color temperatures range from 25 to $45 \mathrm{~K}$ for GF 17 and from 30 to $45 \mathrm{~K}$ for GF 20 . In GF 17, the filamentary region is warmer than the main core region, with clump temperatures of $40 \mathrm{~K}$, and interclump temperatures of $\sim 42 \mathrm{~K}$. For GF 20, this difference is less evident: the temperatures of the clumps within the filamentary region of GF 20 exhibit the same temperature (roughly 31-33 K) of the main core region. The interclump region within the filament is marginally warmer, at $\sim 35 \mathrm{~K}$. Peaks of 43 to $46 \mathrm{~K}$ occur toward the $\mathrm{T}$ Tauri stars near the main core region of GF 20 (see Fig. 6), but care must be taken in interpreting these values, as a steep dust temperature gradient along the line of sight would be expected toward a bright point source within the cloud, invalidating the simple homogeneous model adopted here. These two stars are hot sources seen in the $60 \mu \mathrm{m}$ image which have produced unphysical depressions in the $100 \mu \mathrm{m}$ optical depth image. Away from the central regions of GF 17 and GF 20, the color temperature of the dust emiting at $60 \mu \mathrm{m}$ and $100 \mu \mathrm{m}$ smoothly rises to a maximum of about $45 \mathrm{~K}$, at the optical edges of the clouds.

The highest gas temperatures (given by the $\mathrm{CO}$ antenna temperature) in GF 17 and GF 20 are $12.5 \mathrm{~K}$ and $14.1 \mathrm{~K}$, respectively. Hence, dust temperatures appear to be high enough to heat the gas to those temperatures. However, our dust temperature images show that GF 17 and GF 20 are clearly limb-brightened. This means that the highest gas temperatures occur where the dust temperatures are the lowest, i.e. in the central, denser regions of the clouds. Consequently, the CO must be heated by a source of energy other than grain collisions in the bulk of the clouds. Together with the lack of young stellar objects embedded in GF 17 and GF 20, this seems to indicate that these clouds are heated externally.

The derived $100 \mu \mathrm{m}$ optical depths typically range from $2 \times 10^{-6}$ to $1.8 \times 10^{-5}$ within GF 17 and from $3 \times 10^{-6}$ to $3.8 \times 10^{-5}$ in GF 20 . These values are typically an order of magnitude smaller, and span a narrower range than the values derived by Jarrett et al. (1989) for the $\rho$ Oph cloud, and by Wood et al. (1994) for a sample of 43 clouds with $A_{V} \geq 2 \mathrm{mag}$, using the same method. The lower dynamic range exhibited by $\tau_{100}$ over GF 17 and GF 20 suggests that the grain population responsible for the $100 \mu \mathrm{m}$ emission is likely to be unheated over much of the interior of the clouds, implying that we are probing the edges of GF 17 and GF 20 and not their cold, innermost regions.

\subsection{Dust mass}

To calculate the mass of dust in GF 17 and GF 20 we calculate the mass column density of dust grains at $100 \mu \mathrm{m}$, $\sigma_{\mathrm{d}}$, as

$\sigma_{\mathrm{d}}=\frac{4}{3}\left(\frac{a \rho}{Q_{100}}\right) \tau_{100}\left(\mathrm{~g} \mathrm{~cm}^{-2}\right)$,

where $\rho$ is the grain density, $Q_{100}$ is the emission efficiency at $100 \mu \mathrm{m}$, and where we have assumed that a line of sight 

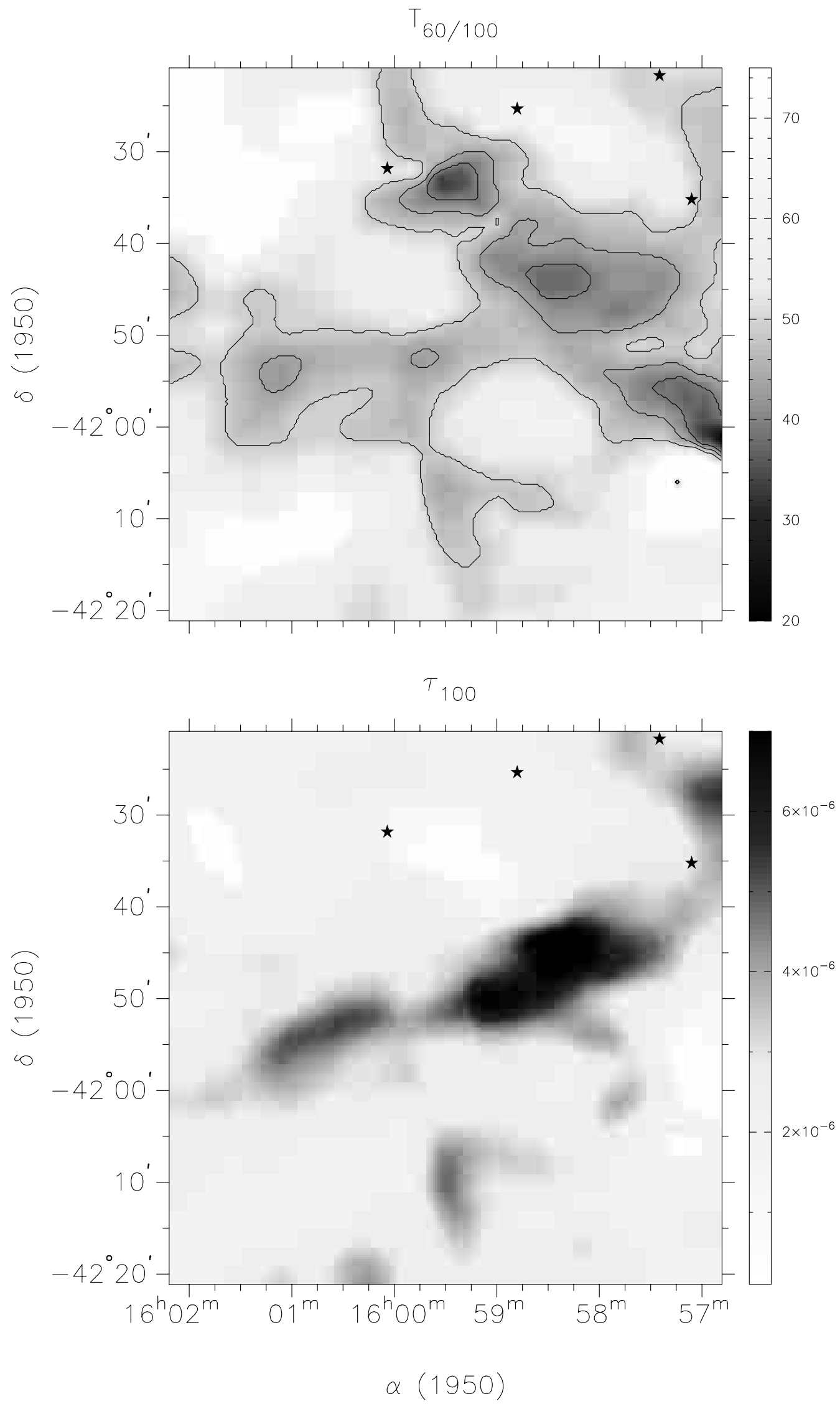

Fig. 5. $T_{60 / 100}$ and $\tau_{100}$ images obtained toward GF 17. Filled stars show the positions of T Tauri stars associated with GF 17 . The temperature scale is degrees Kelvin. Notice the limb brightening in GF 17, with $T_{60 / 100}$ increasing from the inner regions toward the edges of the cloud: for clarity, we plot contours at $35 \mathrm{~K}$ (the innermost contour), $40 \mathrm{~K}$, and $46 \mathrm{~K}$ (the outermost contour). 

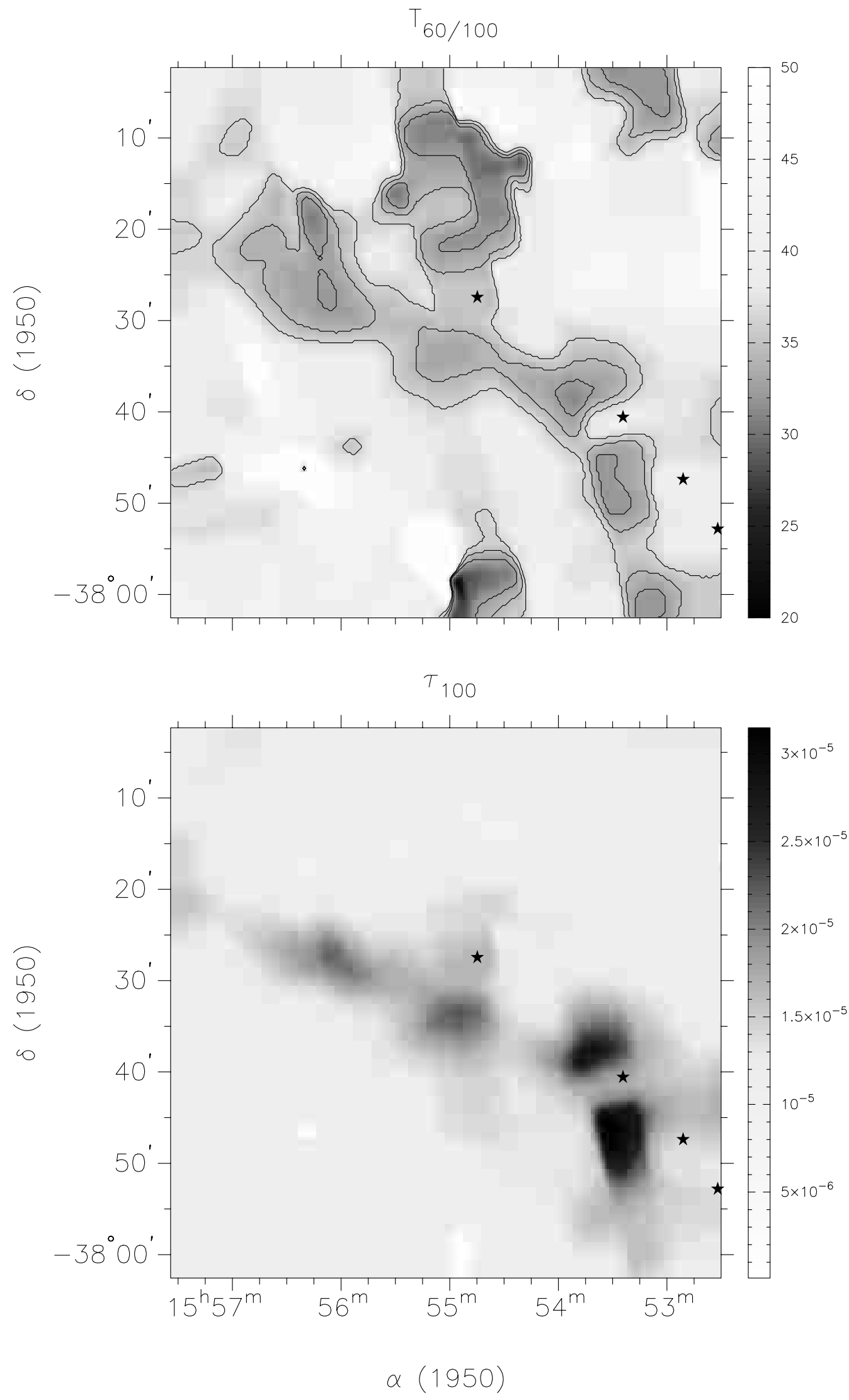

Fig. 6. $T_{60 / 100}$ and $\tau_{100}$ images obtained toward GF 20. Filled stars show the positions of T Tauri stars associated with GF 20. The temperature scale is degrees Kelvin. Notice the limb brightening in GF 20, with $T_{60 / 100}$ increasing from the inner regions toward the edges of the cloud: for clarity, we plot contours at $32 \mathrm{~K}$ (the innermost contour), $35 \mathrm{~K}$, and $38 \mathrm{~K}$ (the outermost contour). 
with column density $N_{\mathrm{g}}$ of grains having radius $a$, has an optical depth at $100 \mu \mathrm{m}$ given by (Hildebrand 1983)

$\tau_{\lambda} \simeq \pi<a>^{2} Q_{\lambda} N_{\mathrm{g}}$.

Using an average value for $\left(a \rho / Q_{100}\right)$ of $3.2 \times\left(1000 / \lambda_{\mu \mathrm{m}}\right)$ for a mixture of graphite and silicate grains (Hildebrand 1983), we have

$\sigma_{\mathrm{d}}=4.17 \times 10^{-2} \tau_{100}\left(\mathrm{~g} \mathrm{~cm}^{-2}\right)$.

Integrating the $\tau_{100}$ images over the area of each cloud (defined as the area of the contour level equal to $20 \%$ of the peak $100 \mu \mathrm{m}$ opacity in the clouds), we obtain an estimate of the dust mass for GF 17 and GF 20. We find total dust masses of about $2.2 \times 10^{-3} M_{\odot}$ and $5.0 \times 10^{-3} M_{\odot}$ for GF 17 and GF 20, respectively. These values decrease to $6.1 \times 10^{-4} M_{\odot}$ and $1.6 \times 10^{-3} M_{\odot}$, respectively, if one considers only the regions covered by our molecular line maps. This would imply gas-to-dust ratios much higher than the typical gas-to-dust ratio of $\sim 100$ in interstellar clouds. However, the derived gas-to-dust ratios for GF 17 and GF 20 are consistent with a true gas-to-dust ratio of $\sim 100$, if we conclude that less than $\sim 1 \%$ of the dust is responsible for the far-infrared emission detected with IRAS. In fact, one should keep in mind that much of the dust in a typical dark cloud is largely undetected by IRAS because it is colder than $\sim 20 \mathrm{~K}$. Owing to the essentially exponential dependence of the infrared emission from such regions, the emission from the cold, high-extinction cores of molecular clouds without massive star formation may be essentially invisible to IRAS.

\subsection{Correlations between gas and dust}

Figures 7 and 8 present ${ }^{13} \mathrm{CO}$ integrated emission maps toward GF 17 and GF 20. The molecular gas emission is not uniformly distributed within the clouds. Instead, the emission is seen to arise in chains of condensations strung along their lengths, in a periodic fashion, giving these clouds an overall highly fragmented appearance. We find a remarkably good agreement between our $100 \mu \mathrm{m}$ optical depth images and our ${ }^{13} \mathrm{CO}$ integrated emission maps. Our optical depth images of GF 17 and GF 20 reproduce the filamentary morphology seen in our ${ }^{13} \mathrm{CO}$ maps. In particular, we see that the $100 \mu \mathrm{m}$ optical depth and ${ }^{13} \mathrm{CO}$ emission images have identified the dense cores within each cloud with remarkable agreement, suggesting that the gas-to-dust ratio is nearly constant throughout these clouds.

\subsubsection{Far-infrared emission as a tracer of gas column density}

Figure 9 presents a point by point comparison of the $100 \mu \mathrm{m}$ optical depth and ${ }^{13} \mathrm{CO}$ integrated emission, $I_{13}$, smoothed to the spatial resolution of IRAS at $100 \mu \mathrm{m}$, for GF 17 (top) and GF 20 (bottom), respectively. In both cases, filled circles refer to observations toward the filamentary region, and empty circles to observations within the main core region. In the case of GF 17 , there is no trend (linear correlation coefficient $r \sim 0.01$ ) of dust $100 \mu \mathrm{m}$ optical depth with ${ }^{13} \mathrm{CO}$ integrated emission within the main core region. However, we find a strong correlation $(r \sim 0.85)$ for the filamentary region. A leastsquares fit to the data within this later region yields

$\tau_{100}=(1.52 \pm 0.26) \times 10^{-6}+(1.28 \pm 0.10) \times 10^{-6} I_{13}$.

For GF 20, a different behaviour is obtained: considering only the data points within the main core region, a strong linear dependence $(r \sim 0.73)$ is found between the dust $100 \mu \mathrm{m}$ optical depth and the ${ }^{13} \mathrm{CO}$ integrated intensity. For the filamentary region, a least-squares fit returns

$\tau_{100}=(5.54 \pm 0.88) \times 10^{-6}+(3.17 \pm 0.46) \times 10^{-6} I_{13}$,

with a correlation coefficient of 0.59 . Note that for the filamentary region of GF 20 the best-fit relation has considerable more scatter than the corresponding relation for GF 17. Although a correlation between $100 \mu \mathrm{m}$ optical depth and ${ }^{13} \mathrm{CO}$ integrated intensity is found in both GF 17 and GF 20, the slopes of these relations are different, with the slope in the gas/dust relation for GF 20 being $\sim 2.5$ times larger than that for GF 17. Also, the $y$-intercept in GF 20 is much larger than that for GF 17, indicating that for GF 20 there is substantial $100 \mu \mathrm{m}$ emission in directions where the integrated ${ }^{13} \mathrm{CO}$ intensity is nearly zero. This may indicate that the background was improperly removed or that the ${ }^{13} \mathrm{CO}$ emission is not tracing the entire column density of gas. Since the ${ }^{13} \mathrm{CO}$ is expected to be destroyed at extinctions less than about $A_{V}=0.5 \mathrm{mag}$, it is reasonable that there be dust and infrared emission where molecular emission cannot be detected. Another feature observed in the correlations shown in Fig. 9 is the significant number of positions (especially in GF 17 ) that lie above the fitted lines at large ${ }^{13} \mathrm{CO}$ integrated emission. Though all lines of sight are optically thin at $100 \mu \mathrm{m}$, not all lines of sight are expected to be thin in ${ }^{13} \mathrm{CO}$ emission, especially toward the denser parts of GF 17 and GF 20. Thus, the scatter of points above the line at large $\left(>3 \mathrm{~K} \mathrm{~km} \mathrm{~s}^{-1}\right){ }^{13} \mathrm{CO}$ integrated emission may indicate that the ${ }^{13} \mathrm{CO}$ emission is saturated, leading to a underestimation of the gas column density therein.

A point by point comparison of the $100 \mu \mathrm{m}$ optical depth and $\mathrm{C}^{18} \mathrm{O}$ integrated intensity, $I_{18}$, smoothed to the spatial resolution of IRAS at $100 \mu \mathrm{m}$, is shown in Fig. 10. For GF 17, the $100 \mu \mathrm{m}$ optical depth clearly increases with increasing $\mathrm{C}^{18} \mathrm{O}$ integrated intensity. Considering all data points, we find a very strong correlation between these two quantities. A least-squares fit yields

$\tau_{100}=(2.24 \pm 0.48) \times 10^{-6}+(1.19 \pm 0.09) \times 10^{-5} I_{18},(12)$

with a correlation coefficient of 0.85 . For GF 20, the linear fit to all data points yields a much poorer $(r=0.46)$ linear correlation

$\tau_{100}=(1.05 \pm 0.08) \times 10^{-5}+(1.09 \pm 0.25) \times 10^{-5} I_{18},(13)$

where we note that very few (only six) data points refer to the main core region of GF 20. However, the slope in 
GF17 filamentary region

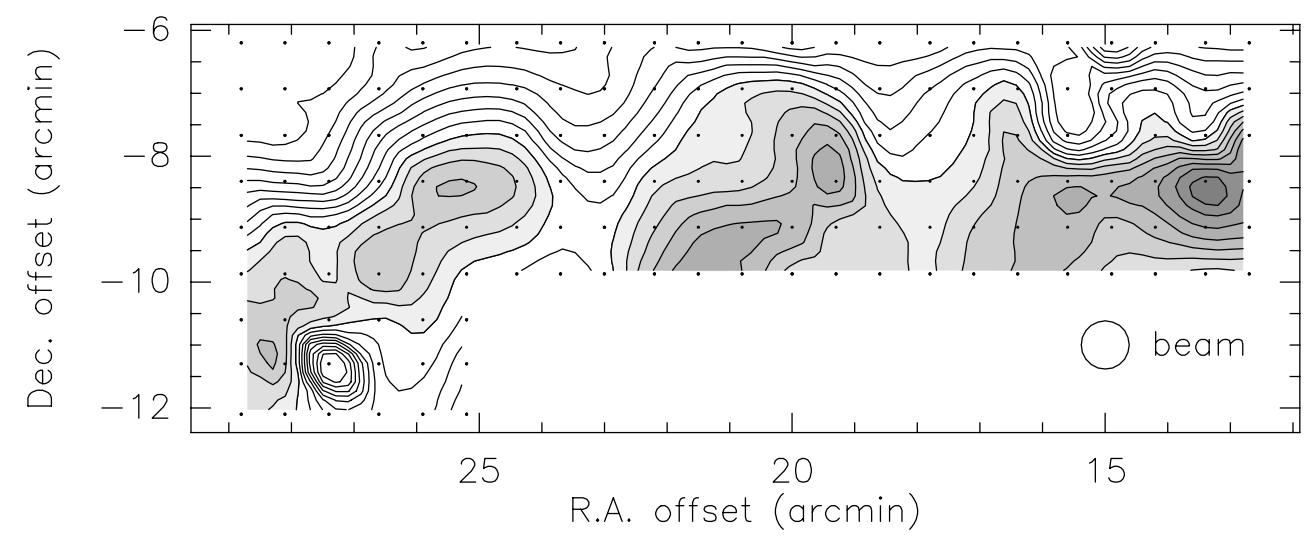

GF17 main core region

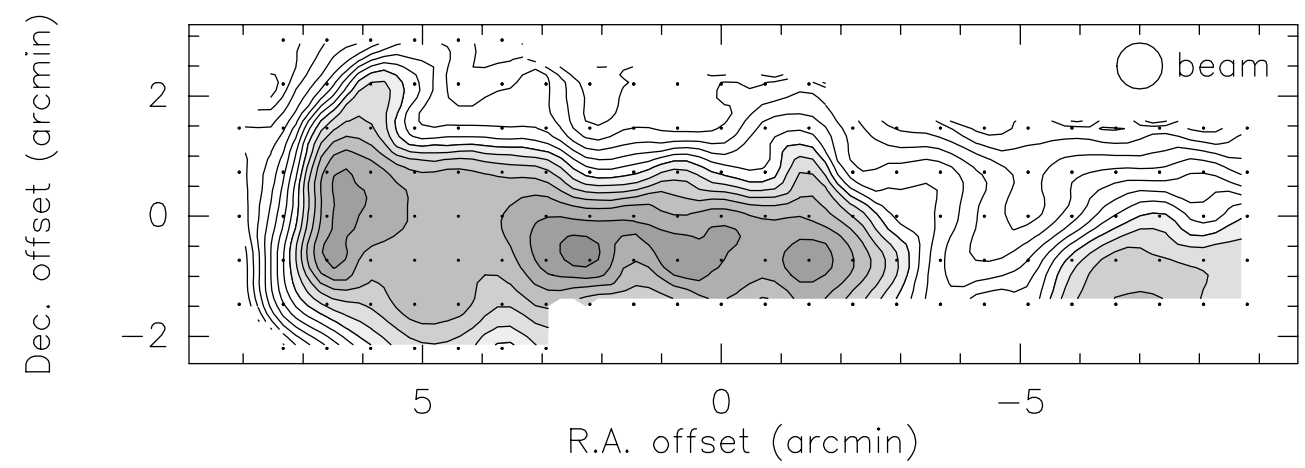

Fig. 7. ${ }^{13} \mathrm{CO}$ integrated emission map toward GF 17. Top panel: contour levels start at $1 \mathrm{~K} \mathrm{~km} \mathrm{~s}^{-1}$ in steps of $0.3 \mathrm{~K} \mathrm{~km} \mathrm{~s}^{-1}$, and shaded contours start at $3.4 \mathrm{~K} \mathrm{~km} \mathrm{~s}^{-1}$. Bottom panel: contour levels start at $3.6 \mathrm{~K} \mathrm{~km} \mathrm{~s}^{-1}$ in steps of $0.3 \mathrm{~K} \mathrm{~km} \mathrm{~s}^{-1}$, shaded contours start at $6.0 \mathrm{~K} \mathrm{~km} \mathrm{~s}^{-1}$. The $(0,0)$ position corresponds to $\mathrm{RA}=16^{\mathrm{h}} 01^{\mathrm{m}} 48.6^{\mathrm{s}}$, Dec $=-41^{\circ} 51^{\prime} 21^{\prime \prime}(\mathrm{J} 2000)$.

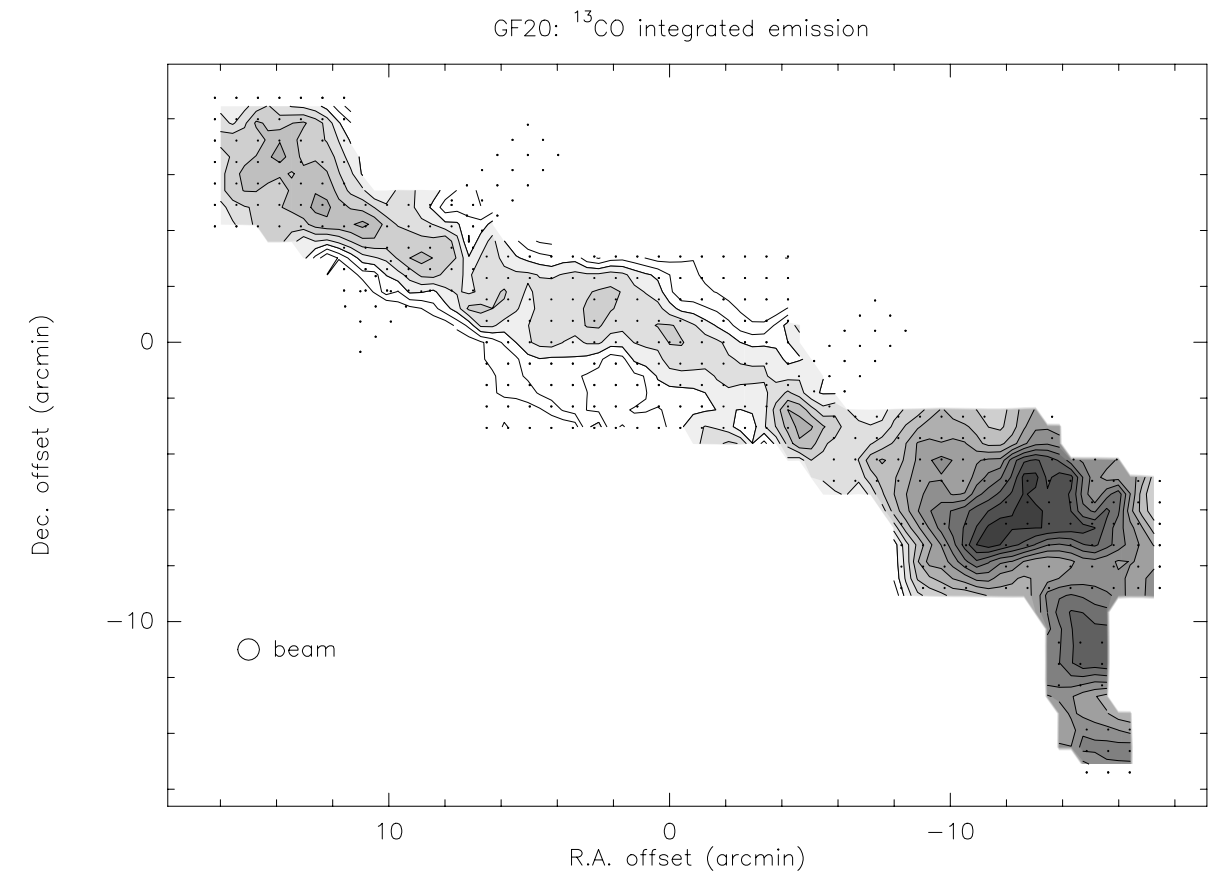

Fig. 8. ${ }^{13} \mathrm{CO}$ integrated emission map toward GF 20. Contour levels range from $1 \mathrm{~K} \mathrm{~km} \mathrm{~s}^{-1}$ to $7.5 \mathrm{~K} \mathrm{~km} \mathrm{~s}^{-1}$ in steps of $0.5 \mathrm{~K} \mathrm{~km} \mathrm{~s}^{-1}$. Shaded contours start at $2 \mathrm{~K} \mathrm{~km} \mathrm{~s}^{-1}$. The $(0,0)$ position corresponds to $\mathrm{RA}=15^{\mathrm{h}} 58^{\mathrm{m}} 00.0^{\mathrm{s}}$, Dec $=-37^{\circ} 42^{\prime} 47^{\prime \prime}$ (J2000). 

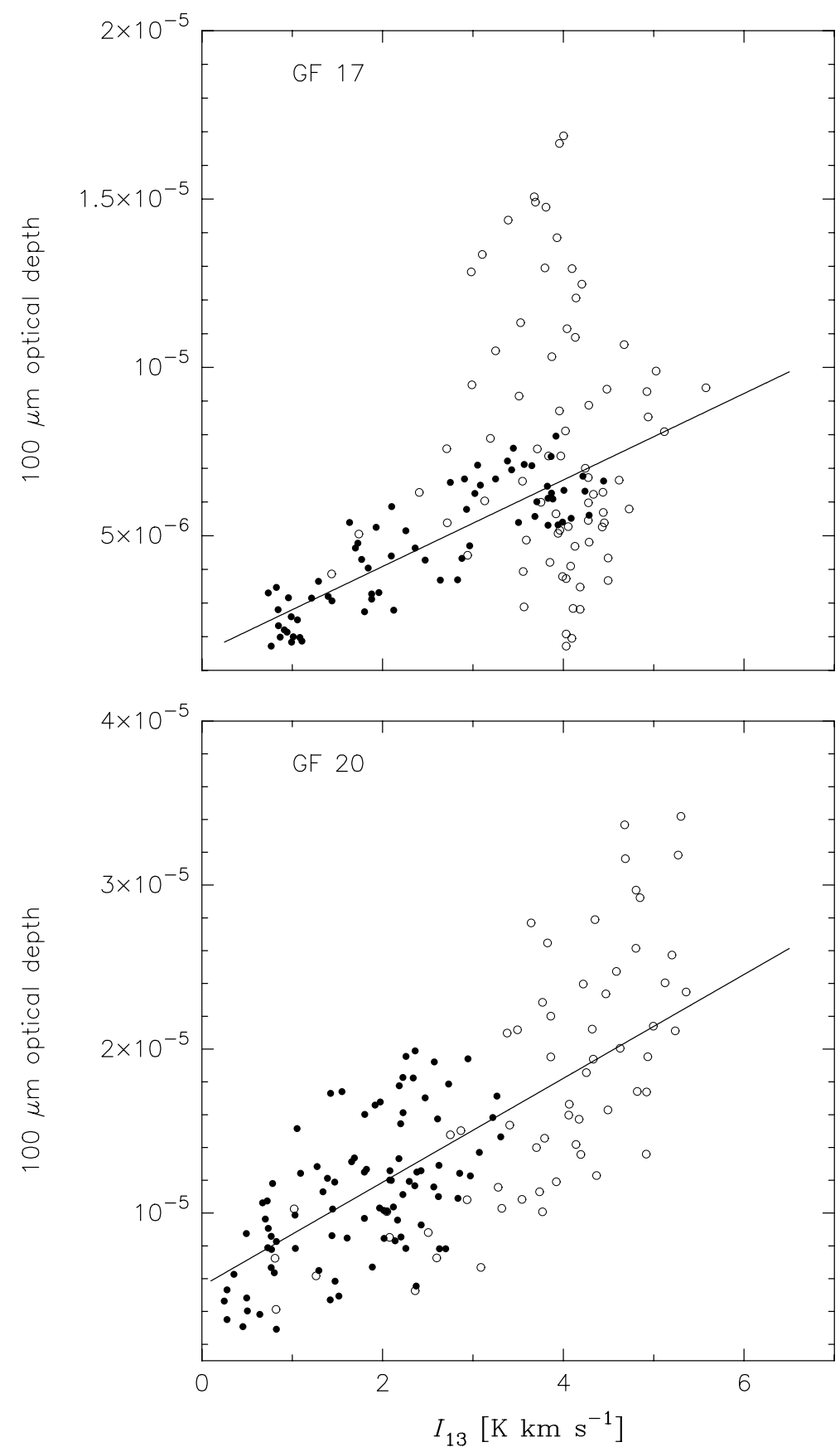

Fig. 9. A point by point comparison of the derived $100 \mu \mathrm{m}$ optical depth to the ${ }^{13} \mathrm{CO}$ integrated intensity in GF 17 (top) and GF 20 (bottom). Filled and empty circles refer to observations toward the filamentary region and the main core region, respectively. The straight lines are least-squares fits to the data obtained toward the filamentary regions only (see text).

GF 20 is similar to the one found for GF 17 . This seems to indicate that additional $\mathrm{C}^{18} \mathrm{O}$ data within the main core region of GF 20 will improve the $\tau_{100}$ vs. $I_{18}$ relation. Hence, despite the larger scatter, we believe that the dust $100 \mu \mathrm{m}$ optical depth and the gas column density in GF 20 are well correlated.

While the dust column density (essentially given by the dust $100 \mu \mathrm{m}$ optical depth) only traces the depth of dust emission, the gas column density (given by the $\mathrm{C}^{18} \mathrm{O}$ integrated intensity) is the true value for the clouds, since the tracer molecule is optically thin. However, the fact that the dust column density is well correlated with the gas column density throughout GF 17 and GF 20 implies that the grains responsible for the 60 and $100 \mu \mathrm{m}$ emission are well mixed with the gas and are heated by a radiation field that impinges these clouds in a relatively uniform fashion. The morphological similarities between the $100 \mu \mathrm{m}$ optical depth images and the $\mathrm{C}^{18} \mathrm{O}$ integrated maps (Moreira \& Yun 2002) of GF 17 and GF 20 support this idea. The good agreement between dust optical depth 

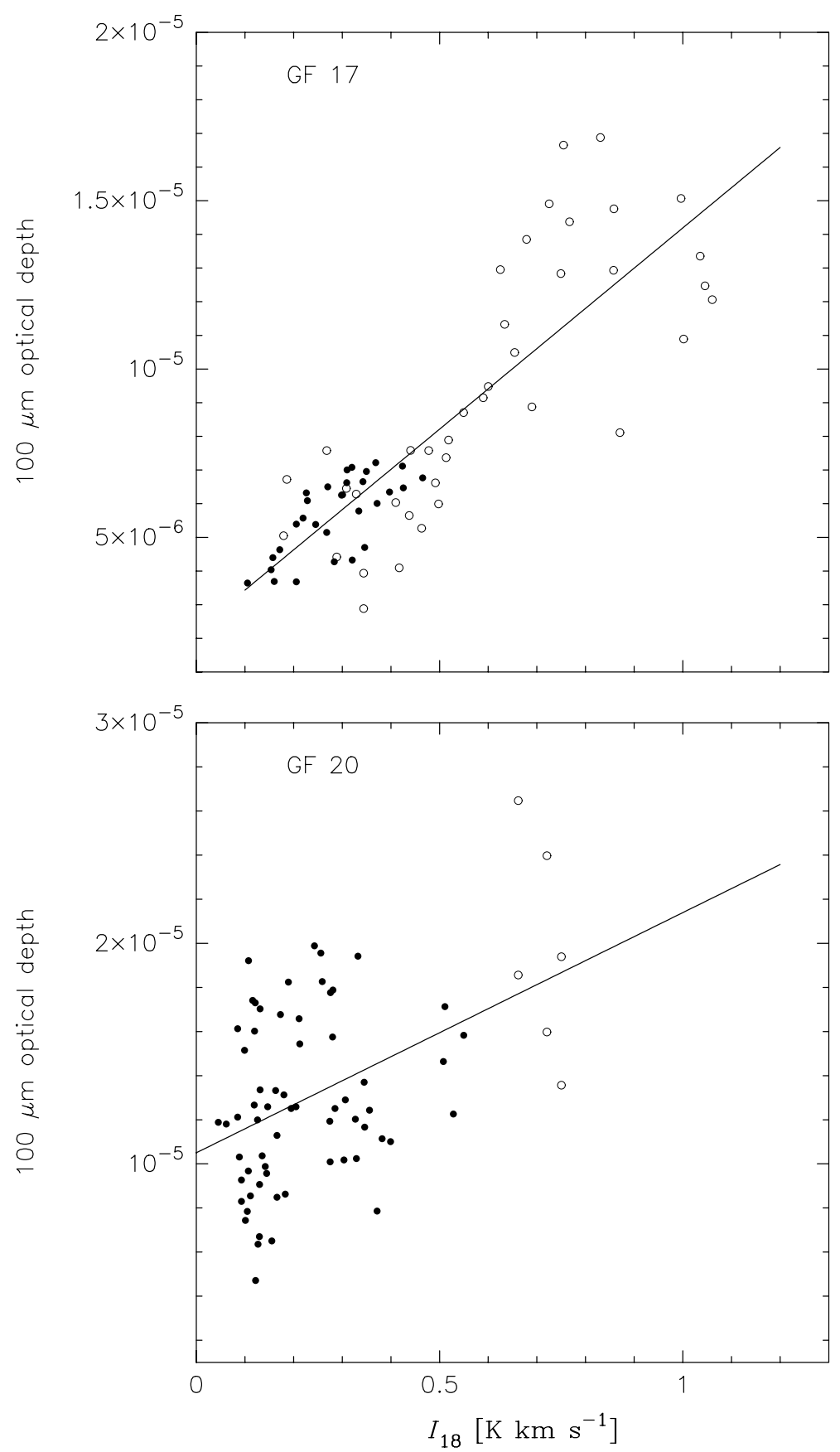

Fig. 10. A point by point comparison of the derived $100 \mu \mathrm{m}$ optical depth to the $\mathrm{C}^{18} \mathrm{O}$ integrated emission in GF 17 (top) and GF 20 (bottom). Filled and empty circles refer to observations toward the filamentary region and the main core region, respectively. The straight lines are least-squares fits to all the data points.

and $\mathrm{C}^{18} \mathrm{O}$ integrated emission in GF 17 and GF 20 then suggests that the infrared emission must originate from a substantial depth in the clouds. This is consistent with the globular nature of GF 17 and GF 20, where we expect the individual dense cores, connected by lower density material, to be more easily exposed to the local radiation field.

We thus conclude that far-infrared dust emission can reliably be used as a gas column density tracer in GF 17 and GF 20.

\subsubsection{Dust and gas temperatures}

A point by point comparison of the dust temperature and gas column density in GF 17 and GF 20 is shown in Fig. 11 revealing an anticorrelation between these two quantities. The spatial resolutions of both data sets were degraded to match the IRAS $100 \mu \mathrm{m}$ beam size. The temperature of the dust varies from 33 to $51 \mathrm{~K}$ in GF 17 , and from 31 to $42 \mathrm{~K}$ in GF 20. Hence, the emitting dust in both clouds 

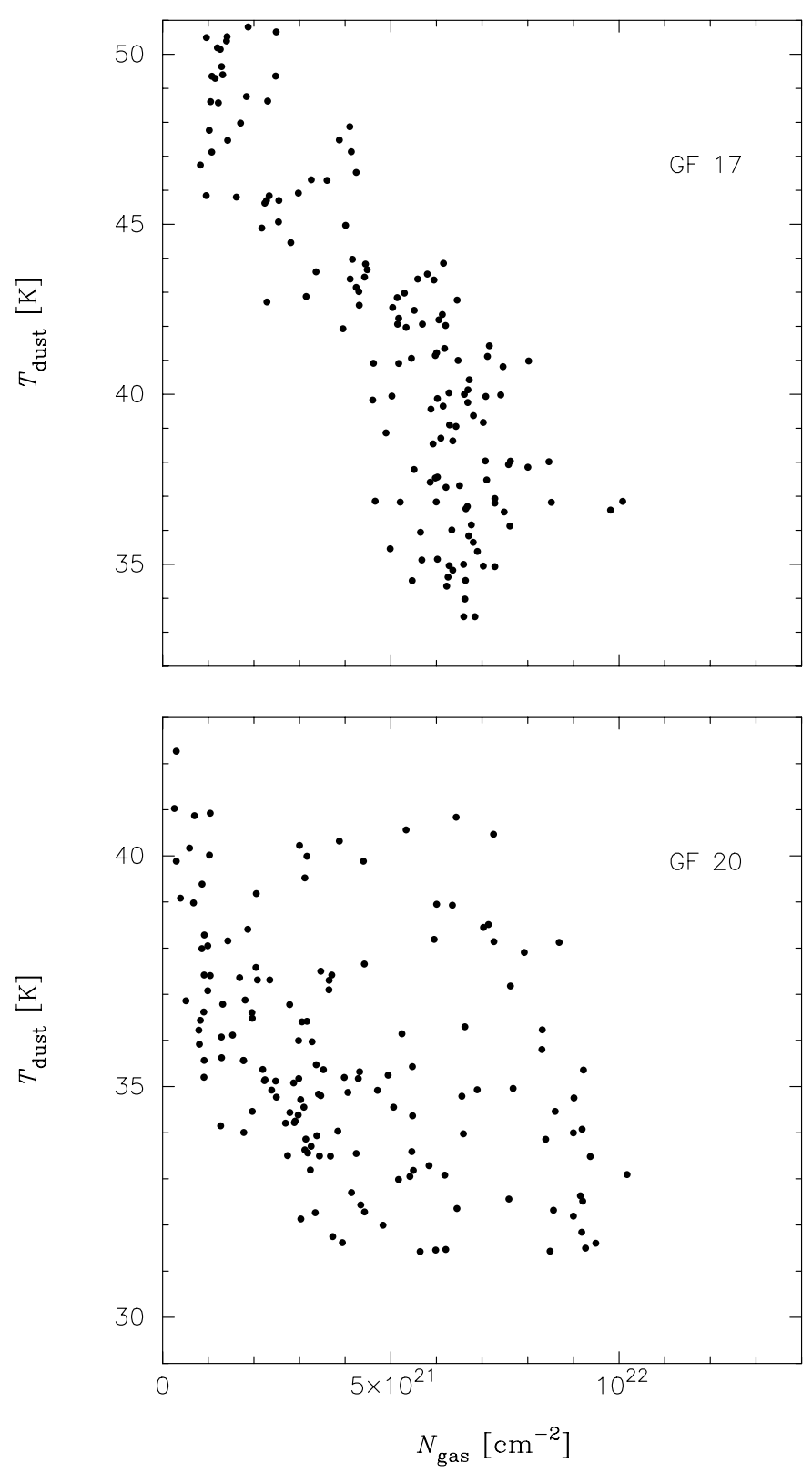

Fig. 11. A point by point comparison of the derived dust temperature to the total column density of gas in GF 17 (top) and GF 20 (bottom). Notice that the higher dust temperatures are found towards lower gas column densities.

is substantially hotter than the gas. However, one must be cautious in interpreting these results, since, as noted above, the derived dust temperature is always weighted toward the warmest dust along the line of sight, and dust as cold as the gas will be overwhelmed by the warmer dust and will be unobservable. This explains the fact that the temperatures we calculate (see Table 1) even in the denser, starless main core regions, are significantly higher than $\sim 20 \mathrm{~K}$, while the true gas temperatures (as derived by the ${ }^{12} \mathrm{CO}$ radiation temperatures) are typically below $20 \mathrm{~K}$. Despite these potential difficulties, we believe that the anticorrelation found between the dust temperature and gas column density implies that the dust is warmest where the column densities are smallest. In fact, from the dust temperature maps of GF 17 and GF 20, it is clear that the hotter dust is located at the edges of the clouds. Thus, we find further evidence for GF 17 and GF 20 being externally heated.

\subsection{Evidence for smooth cloud edges}

Our calculated dust optical depths toward GF 17 and GF 20 are small, with typical values of $\tau_{100}$ about a factor of 10 smaller than the corresponding values derived by Wood et al. (1994) for 43 nearby molecular clouds. Consequently, small ( $<1 \mathrm{mag}$ ) values of visual extinction (see Table 1) are obtained for GF 17 and GF 20 by means of Eq. (3), and we remind the reader that all extinctions quoted here are lower limits. In order to estimate the pixelto-pixel (random) errors in $A_{V}$ in each cloud, we examined about 800 pixels within a circular area which appears to have a constant extinction, and obtained a standard deviation in the extinction value of this region of $0.02 \mathrm{mag}$ for both clouds. We use these values as an estimate of the pixel-to-pixel errors in $A_{V}$, but we remind the reader that this error does not include any errors caused by assuming a constant $\beta$.

Lada et al. (1994) showed that the relation between $\sigma_{\text {disp }}$, the dispersion of extinction measurements within a square map pixel, and $A_{V}$, the mean extinction derived for the map pixel, can be used to characterize cloud structure on scales smaller than the resolution of the map (i.e. the size of the map pixels). In the molecular cloud IC 5146, Lada et al. (1994) and Lada et al. (1999) found that both $\sigma_{\text {disp }}$ and the dispersion in the $\sigma_{\text {disp }}-A_{V}$ relation increased in a systematic fashion with increasing $A_{V}$. A similar behaviour for the L977 dark cloud was found by Alves et al. (1998). In order to investigate a $\sigma_{\text {disp }}-A_{V}$ relation for GF 17 and GF 20, we used the $A_{V}$ images generated through Eq. (3). In each $A_{V}$ image, we considered square map pixels (each containing about 400 image pixels) with size similar to the IRAS beam size $\left(300^{\prime \prime}\right)$ at $100 \mu \mathrm{m}$. A mean extinction and a dispersion within each map pixel were derived. The top panels of Figs. 12 and 13 present the $\sigma_{\mathrm{disp}}-A_{V}$ relation for the two clouds, at $300^{\prime \prime}$ spatial resolution. The same trend observed in IC 5146 and L977 is found for GF 17 and GF 20. Both $\sigma_{\text {disp }}$ and the scatter in the $\sigma_{\text {disp }}-A_{V}$ relation increase systematically with $A_{V}$ in GF 17 and GF 20. A least-squares fit over the entire data sets returns the slope of the $\sigma_{\mathrm{disp}}-A_{V}$ relation given by

GF 17: $\quad \sigma_{\text {disp }} / A_{V}=0.18 \pm 0.01$

GF 20: $\sigma_{\text {disp }} / A_{V}=0.34 \pm 0.02$.

These values are similar to $\sigma_{\text {disp }} / A_{V}=(0.21 \pm 0.02)$ and $\sigma_{\text {disp }} / A_{V}=(0.40 \pm 0.01)$ found for IC5146 and L977, respectively, by Alves et al. (1998) and Lada et al. (1999), using near-infrared extinction maps at $90^{\prime \prime}$ spatial filtering. Since the estimated distance to both L977 and IC5146 is $\sim 500 \mathrm{pc}$, their study has a spatial resolution of $0.2 \mathrm{pc}$. 

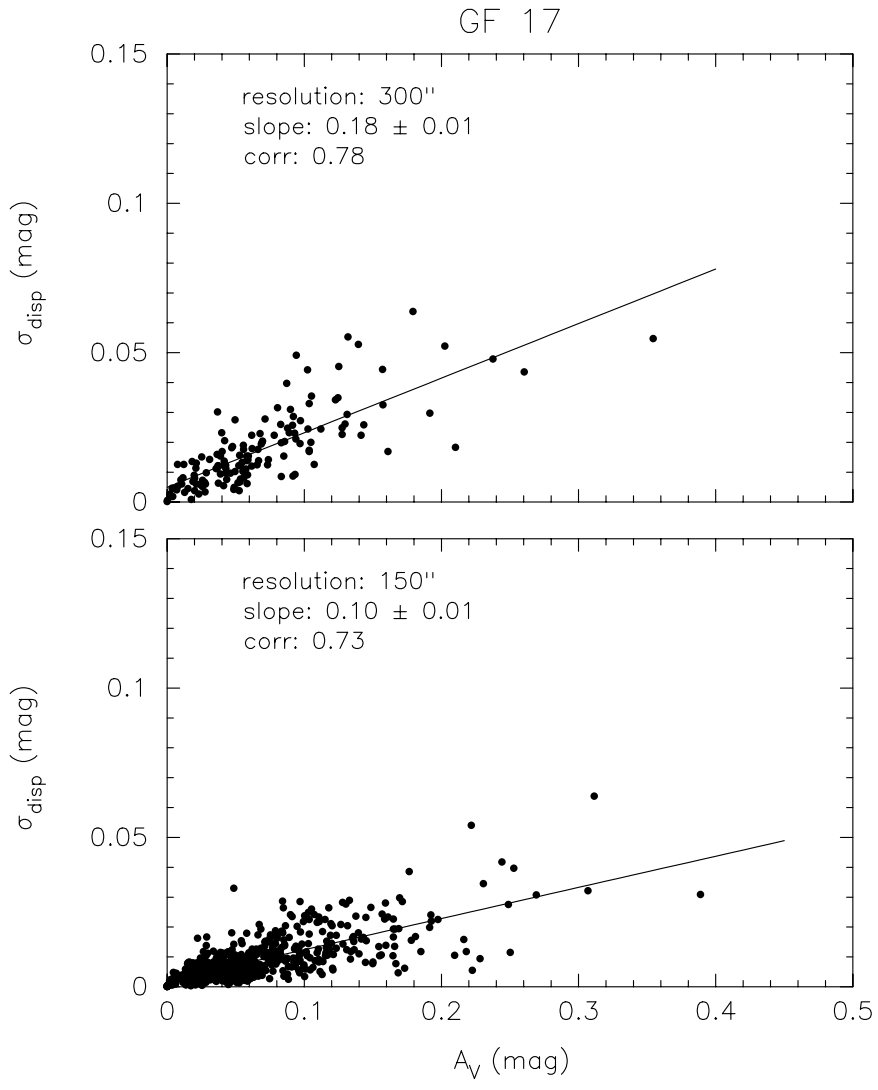

Fig. 12. Top panel: the relation between $\sigma_{\text {disp }}$, the dispersion in the extinction measurements, and $A_{V}$, the mean extinction derived within a $300^{\prime \prime}$ square map pixel for GF 17 . Also plotted is the least-squares linear fit to the data over the entire range of extinctions observed in the cloud. The correlation coefficient is 0.78 . Bottom panel: the same relation but with a spatial filter with angular resolution of $150^{\prime \prime}$. The solid line represents a least-squares linear fit to the data over the entire range of extinctions observed in the cloud. The correlation coefficient is 0.73 . Note the decrease in the slope of the $\sigma-A_{V}$ relation with increasing resolution.

Interestingly, the present IRAS study of GF 17 and GF 20 (at $150 \mathrm{pc}$ ) has the same spatial resolution of $0.2 \mathrm{pc}$. Hence, we are probing structures with similar physical sizes. Also interesting is the fact that from figure 9 of Lada et al. (1999) we note that for extinctions below a few magnitudes (say less than $5 \mathrm{mag}$ ) their $\sigma_{\text {disp }}-A_{V}$ relation is pure noise, while in our study we are sensitive to structure in the same relation but below $A_{V}=1$. Thus, the NIR- and IRAS-based techniques seem to be complementary regarding the $\sigma_{\mathrm{disp}}-A_{V}$ relation.

Lada et al. (1994) have shown that such a relation between $\sigma_{\text {disp }}$ and $A_{V}$ indicate that significant structure must be present down to scales smaller than the resolution of the extinction maps. Thus, the $\sigma_{\mathrm{disp}}-A_{V}$ relations for GF 17 and GF 20 seem to imply the presence of smallscale structure in the exctinctions toward these clouds. Recently, Lada et al. (1999) used Monte Carlo simulations to show that the form and slope of the $\sigma_{\mathrm{disp}}-A_{V}$ relation, and hence most (if not all) of the small-scale variations in the extinction, are due to unresolved gradients
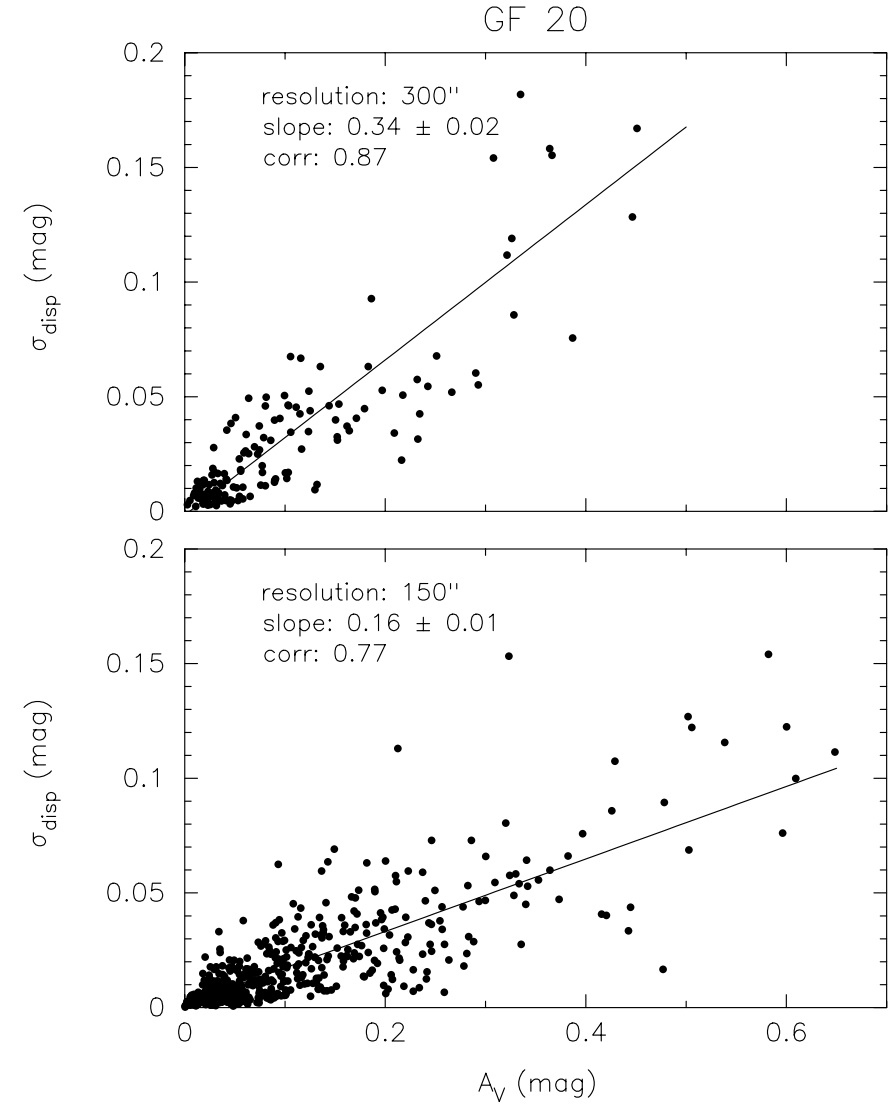

Fig. 13. Top panel: the relation between $\sigma_{\text {disp }}$, the dispersion in the extinction measurements, and $A_{V}$, the mean extinction derived within a $300^{\prime \prime}$ square map pixel for GF 20 . Also plotted is the least-squares linear fit to the data over the entire range of extinctions observed in the cloud. The correlation coefficient is 0.87 . Bottom panel: the same relation but with a spatial filter with angular resolution of $150^{\prime \prime}$. The solid line represents a least-squares linear fit to the data over the entire range of extinctions observed in the cloud. The correlation coefficient is 0.77 . Note the decrease in the slope of the $\sigma-A_{V}$ relation with increasing resolution.

in the dust distribution within IC 5146 and L977. That is to say that smoothly varying density gradients can produce the "fluctuations" observed in extinction studies of filamentary clouds. Although Padoan et al. (1997) found that the form of the observed $\sigma_{\text {disp }}$ versus $A_{V}$ relation in IC5146 is consistent with cloud structure models characterized by supersonic random motions, Lada et al. (1999) note that random spatial fluctuations in the dust distribution could exist (Thoraval et al. 1997), but at a very low level $\left(\sigma_{\text {random }} / A_{V} \ll 25 \%\right.$ at $A_{V} \sim 30 \mathrm{mag}$ ), in addition to the smooth gradients.

Consider Figs. 12 and 13 (bottom panels), where we plot the $\sigma_{\mathrm{disp}}-A_{V}$ relation for both GF 17 and GF 20, but using a spatial filter with angular resolution of $150^{\prime \prime}$, i.e. half the IRAS beamsize at $100 \mu \mathrm{m}$. These diagrams show that when the data are sampled with increased spatial resolution, we obtain a decrease in the slope of the $\sigma_{\text {disp }^{-}}$ $A_{V}$ relation. Also, note that the trend of $\sigma_{\text {disp }}$ increasing with $A_{v}$ appears to be independent of angular resolution. 
This suggests that structural variations in GF 17 and GF 20 are being increasingly resolved out with higher angular resolution. An identical behaviour was found in IC 5146 by Lada et al. (1999), who argue that such behaviour can be accounted for by a smooth, radially decreasing density gradient of the form $\rho(r) \propto r^{-2}$, from 0 to $\sim 20$ mag of visual extinction. In this context, since we are biased toward low $\left(A_{v}<1 \mathrm{mag}\right)$ extinctions, we conclude that the edges of GF 17 and GF 20 are likely to be characterized by a similar smooth density gradient. An investigation is underway on the modelling of the internal structures of GF 17 and GF 20 as self-gravitating cylindrical polytropes, and we defer such discussion to a future paper.

\subsection{Comparison with infrared cirrus clouds and molecular dark clouds}

One of the new phenomena discovered by the IRAS mission is the extensive diffuse infrared emission, strongest at $100 \mu \mathrm{m}$, which has become known as the infrared cirrus. These highly structured extended sources are seen predominantly, but not exclusively, at 60 and $100 \mu \mathrm{m}$ and may originate either in the interplanetary medium, the outer solar system, or the interstellar medium. The infrared cirrus have typical visual extinctions of $\sim 0.2 \mathrm{mag}$ or less and $100 \mu \mathrm{m}$ opacities in the range $3-6 \times 10^{-5}$ (Low et al. 1984). Several studies (Blitz et al. 1984; Weiland et al. 1986; de Vries et al. 1987) have shown that molecular clouds have been found to be associated with infrared cirrus. Thus, it is of interest to compare the far-infrared emission from GF 17 and GF 20 with the more diffuse infrared cirrus clouds. The most useful means of comparison of these clouds is through the ratio of the $100 \mu \mathrm{m}$ intensity, $I_{100}$, versus the column density of hydrogen atoms, $N_{\mathrm{H}}$. In the cirrus clouds, Low et al. (1984) derived values of $I_{100} / N_{\mathrm{H}}$ in the range $0.9-2.8 \mathrm{MJy} \mathrm{sr}^{-1} /\left(10^{20} \mathrm{H} \mathrm{cm}^{-2}\right)$. Other values of $I_{100} / N_{\mathrm{H}}$ of 0.4 to $1.4 \mathrm{MJy} \mathrm{sr}^{-1} /\left(10^{20} \mathrm{H} \mathrm{cm}^{-2}\right)$ were derived by Boulanger et al. (1985), Terebey \& Fich (1986), and Boulanger \& Perault (1988). Values of $I_{100} / N_{\mathrm{H}}$ as high as $1.9 \mathrm{MJy} \mathrm{sr}^{-1} /\left(10^{20} \mathrm{H} \mathrm{cm}^{-2}\right)$ have been found for a number of molecular clouds (Boulanger 1989).

Plots of the $100 \mu \mathrm{m}$ intensity versus molecular hydrogen column density in the edges (i.e., at those locations where the visual extinction is less than say $\sim 2 \mathrm{mag}$ ) of GF 17 and GF 20 are shown in Fig. 14. The spatial resolutions of both data sets were smoothed to the IRAS beam size at $100 \mu \mathrm{m}$. We note that the $100 \mu \mathrm{m}$ intensity follows closely (correlation coefficient of $r \sim 0.78$ ) the gas column density in the vicinity of GF 17 , whereas a rather poor linear trend $(r \sim 0.31)$ is seen for GF 20. From the least-squares fits of $I_{100}$ versus $N_{\text {gas }}$ shown in Fig. 14, we have computed the ratio of $100 \mu \mathrm{m}$ intensity to total molecular hydrogen column density, and we obtain 1.78 and $0.76 \mathrm{MJy} \mathrm{sr}^{-1}$ per $10^{21} \mathrm{~cm}^{-2}$ at the edges of GF 17 and GF 20, respectively. Following Snell et al. (1989), we express these ratios in terms of hydrogen atoms to find values of 0.09 and $0.04 \mathrm{MJy} \mathrm{sr}^{-1}$ per $10^{20} \mathrm{H}$ atoms $\mathrm{cm}^{-2}$ for GF 17 and GF 20, respectively. These values are in excellent agreement with the results for B18 (0.07 $\left.\mathrm{MJy} \mathrm{sr}^{-1} /\left[10^{20} \mathrm{H} \mathrm{cm}^{-2}\right]\right)$, and significantly smaller than those found for the cirrus clouds. Hence, our clouds have lower emission per hydrogen atom than the cirrus clouds.

One can also compare the ratio of 60 to $100 \mu \mathrm{m}$ intensity in our clouds with that found for the cirrus clouds. Low et al. (1984) and Terebey \& Fich (1986) obtained an average $I_{60} / I_{100}$ ratio of 0.20 for the cirrus clouds. In GF 17 and GF 20, we find a systematic decrease of the $I_{60} / I_{100}$ ratio from the edges $\left(I_{60} / I_{100} \sim 0.3\right)$ to the center of the clouds $\left(I_{60} / I_{100} \sim 0.16\right)$. Thus, it seems that GF 17 and GF 20 have similar $I_{60} / I_{100}$ average ratios, and comparable to the corresponding ratio found in cirrus clouds. This is somewhat unexpected because due to their low visual extinctions ( $A_{V} \sim 0.07-0.18 \mathrm{mag}$, Low et al. 1984), the dust in cirrus clouds can be heated to temperatures significantly larger than the typical IRAS dust temperatures (20-25 K) observed in the inner regions of cold dark clouds (Wood et al. 1994). Therefore, the $I_{60} / I_{100}$ ratio appears to be somewhat enhanced in GF 17 and GF 20. However, while the $100 \mu \mathrm{m}$ emission comes from large dust grains in equilibrium with the radiation field, part of the $60 \mu \mathrm{m}$ emission may arise from transiently excited particles (Puget \& Léger 1989). Hence, we can interpret our enhanced $I_{60} / I_{100}$ ratios in GF 17 and GF 20 as the result of an excitation effect where small grains absorb mainly in the UV and consequently are heated only in a shell at the surfaces of GF 17 and GF 20. This is consistent with the fact that our clouds have (1) comparable peak brightness at either 60 or $100 \mu \mathrm{m},(2)$ similar $60-100 \mu \mathrm{m}$ colors, as given by the $I_{60} / I_{100}$ ratios, and (3) similar color morphology, in the sense that they exhibit systematic color variations correlated with the opacity (a decrease by a factor of 2 to 3 from the edges to the center).

Though the IRAS 60 and $100 \mu \mathrm{m}$ bands do not detect the emission from most of the dust in these clouds, these bands do include most of the far-infrared luminosity. We can estimate the total far-infrared luminosities of GF 17 and GF 20 from the $60 \mu \mathrm{m}$ and $100 \mu \mathrm{m}$ images using

$L_{\mathrm{FIR}}=4 \pi d^{2} \int F_{\nu} \mathrm{d} \nu=4 \pi d^{2} \sum F_{\nu} \nu$

where $d$ is the distance to the clouds (taken to be $150 \mathrm{pc}$ ). We obtain total luminosities of $\sim 22 L_{\odot}$ and $\sim 30 L_{\odot}$ for GF 17 and GF 20, respectively. Considering only the regions covered by our molecular line maps, the total luminosities decrease to $\sim 4.5 L_{\odot}$ and $\sim 6.6 L_{\odot}$ for GF 17 and GF 20, respectively. Using the cloud masses derived above, we derive ratios of far-infrared luminosity-to-cloud mass of $0.13 L_{\odot} / M_{\odot}$ and $0.21 L_{\odot} / M_{\odot}$. For comparison, Snell et al. (1989) obtain luminosity-to-mass ratios of $\sim 0.3 L_{\odot} / M_{\odot}$ for B18 and Heiles Cloud 2 in Taurus, Jarrett et al. (1989) derive $\sim 0.6 L_{\odot} / M_{\odot}$ for the $\rho$ Oph dark cloud, and Clemens et al. (1991) find an average of $\sim 0.5 L_{\odot} / M_{\odot}$ for their sample of Bok globules. Note that 

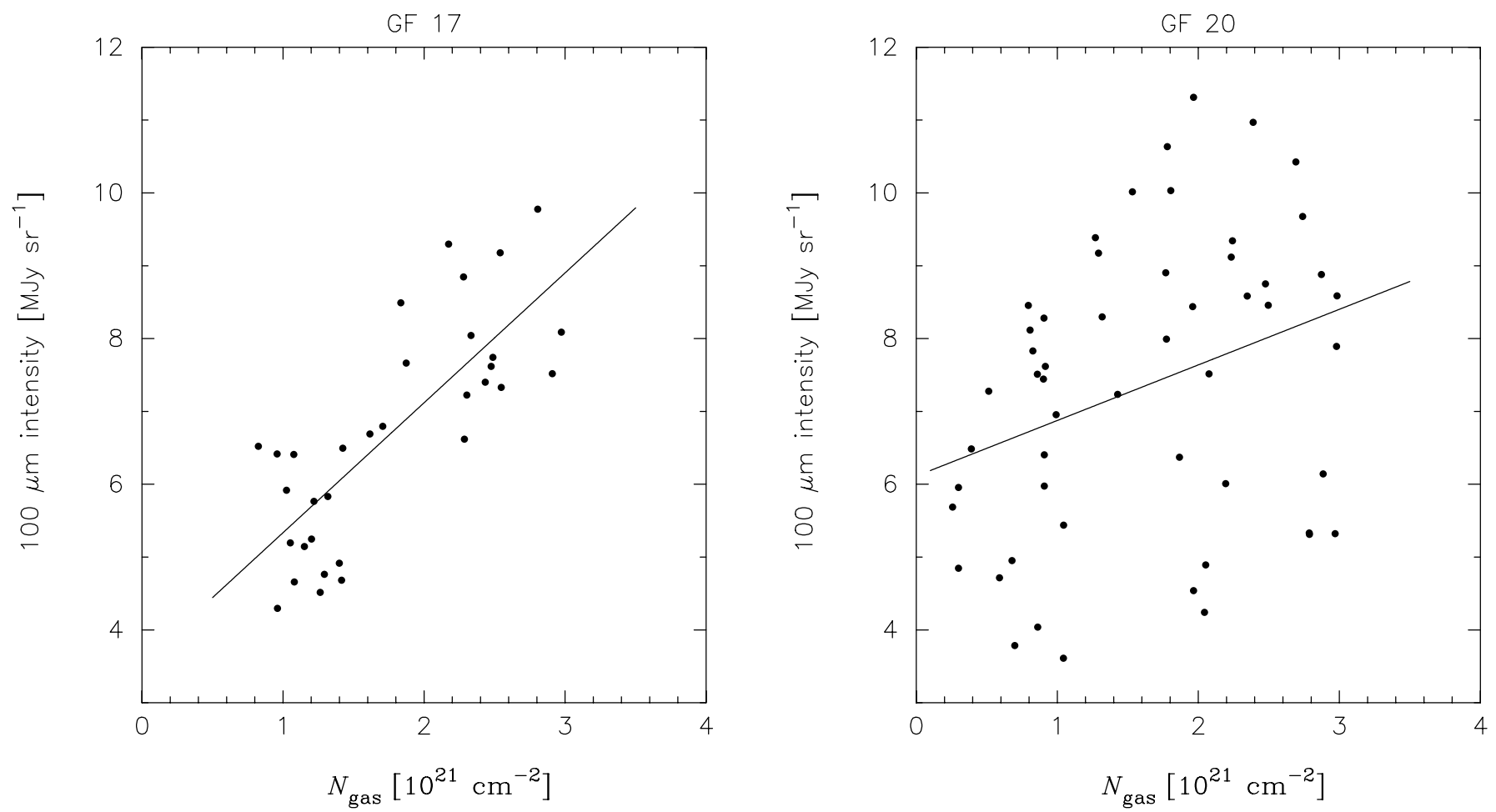

Fig. 14. A point by point comparison of the $100 \mu \mathrm{m}$ intensity to the total molecular hydrogen column density at the edges $\left(A_{V}<2 \mathrm{mag}\right)$ of GF 17 and GF 20. Both data sets were smoothed to the spatial resolution of IRAS at $100 \mu \mathrm{m}$. The solid lines represent least-squares linear fits to the data.

the luminosity-to-cloud mass ratio is a quantity which is independent of distance. The average value for the inner Galactic disk (excluding the galactic center) is $2.8 L_{\odot} / M_{\odot}$ (Scoville \& Good 1987). Thus, GF 17 and GF 20 have smaller luminosity-to-mass ratios than the average Bok globule or the $\rho$ Oph dark cloud, but comparable to the luminosity-to-mass ratios found in Taurus.

\subsection{Velocity structure of the clouds}

The kinematics of a molecular cloud reflects the motions which brought the gas to its current configuration, and can be used to characterize the cloud's evolution. Kinematic signatures in a cloud can result from a variety of phenomena like expanding H II regions, powerfull stellar winds, supernova explosions, outflows, magnetic fields, or even from simple solid-body rotation or galactic shear. For example, the morphology of the $\rho$ Oph complex (Vrba 1977; Loren 1989) suggests that both shocks and magnetic fields are the main mechanisms responsible for the elongation of the dark clouds L1709, L1755, L1729, and L1689N. These clouds are long filaments extending from the starforming cores in $\rho \mathrm{Oph}$, and are aligned along a direction pointing toward the Upper-Scorpius (hereafter USco) subgroup of the Sco OB2 association (Loren 1989). External forces, such as an expanding supernova remnant or H II shell, applied to a gas complex can accelerate different clump masses at different rates. Differential acceleration can stretch a cloud into an elongated filament with the most massive component closest to the source of the external force. As a result, a velocity gradient is expected to appear along the filament axis. On the other hand, if different mass elements along a filament's length have the same $V_{\mathrm{LSR}}$, then it is evidence for there being no component of external force along the line of sight. Finally, while any differences in $V_{\mathrm{LSR}}$ from one end of a filament to the other are most likely not the result of rotation, transverse gradients are more likely to be the result of large-scale rotation (Goodman et al. 1993). Thus, studies of the velocity fields within molecular clouds are of crucial importance in order to characterize the dynamical state of the clouds.

The extensive velocity information contained in spectral-line maps provides the opportunity to analyse motions in molecular clouds carefully, and thus to estimate accurately the magnitude and direction of the velocity gradients, if present. The $\mathrm{CO}$ and ${ }^{13} \mathrm{CO}$ lines are a useful probe of the large-scale velocity field in a cloud because of its widespread detectability, but are not an unbiased probe in all cases, due to opacity effects. In the case of GF 20, we have decided to use the CO line as a probe because the lines are gaussian in shape and narrow $\left(\Delta v \sim 0.9 \mathrm{~km} \mathrm{~s}^{-1}\right)$. For GF 17, we selected the ${ }^{13} \mathrm{CO}$ line because the $\mathrm{CO}$ line was found to be very assymetric and broad $\left(\Delta v \sim 1.3 \mathrm{~km} \mathrm{~s}^{-1}\right)$ toward most lines-of-sight. Nevertheless, it is extremely unlikely that our velocity gradient calculations will be significantly affected by opacity effects because narrow linewidths do not allow large errors in velocity, even for lines-of-sight with large optical 
GF 17

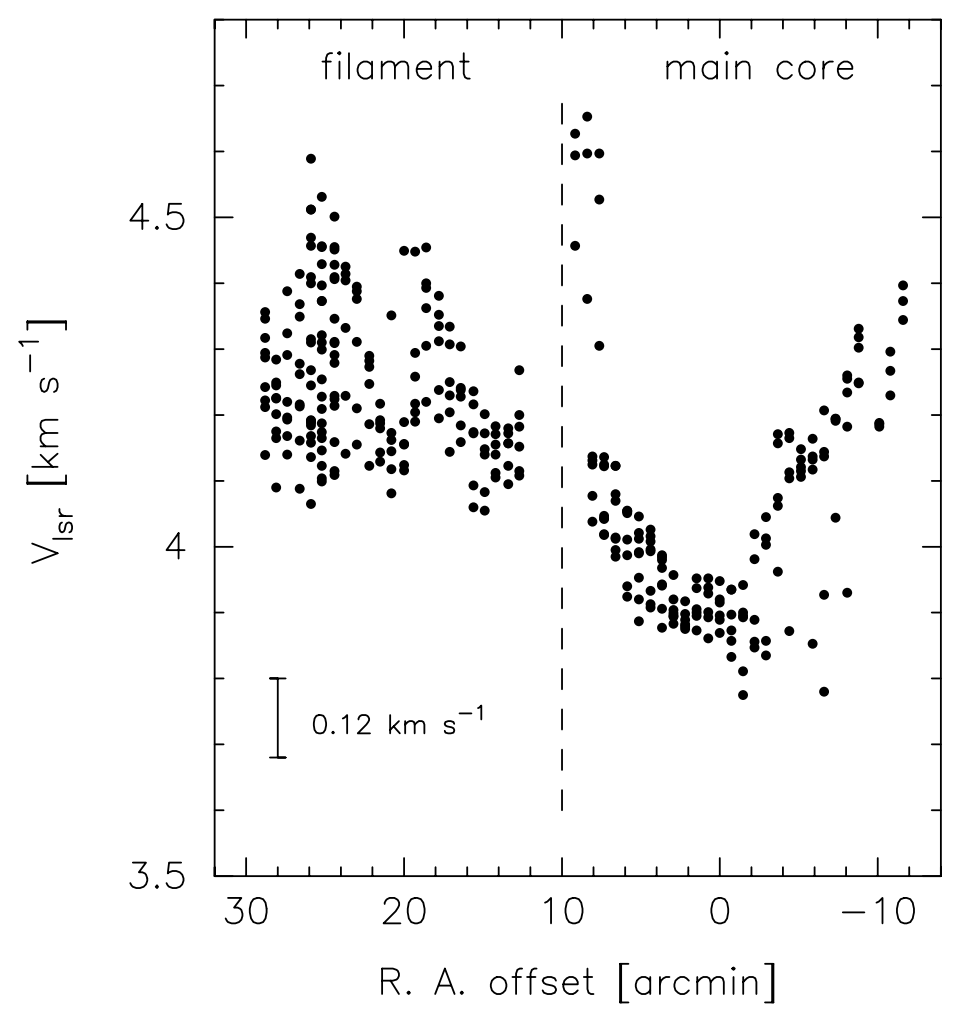

GF 20

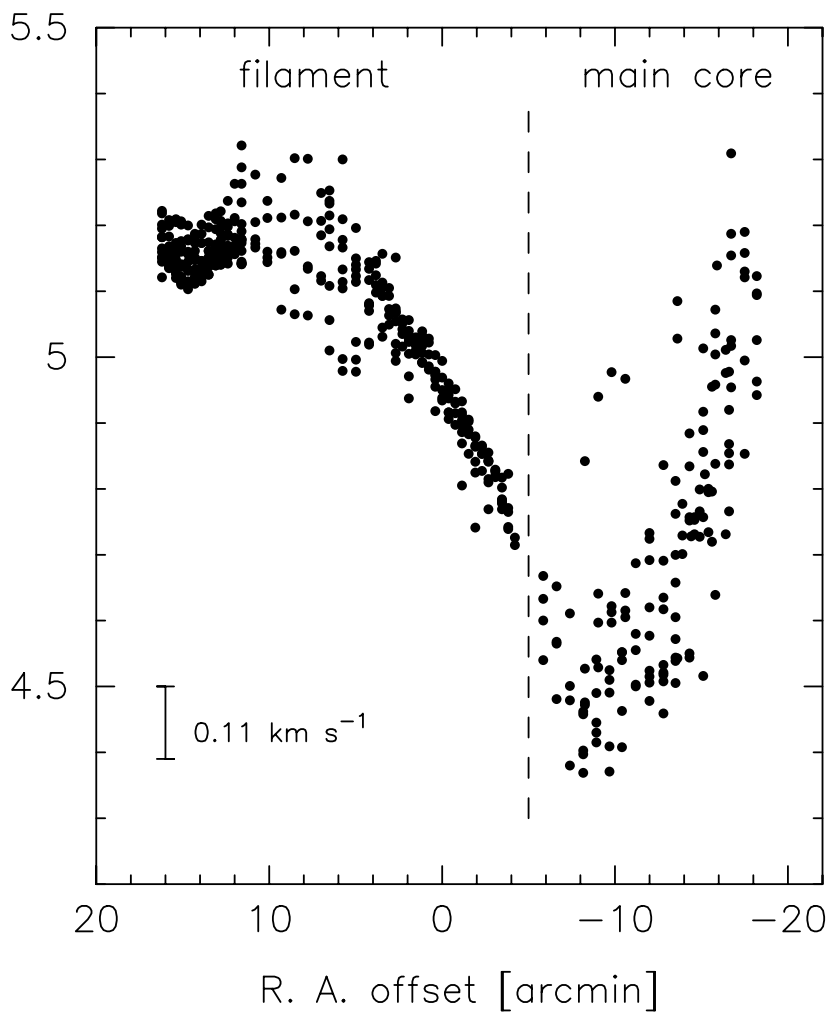

Fig. 15. Left panel: variation of the LSR central velocity of the ${ }^{13} \mathrm{CO}$ line as function of right ascension offset in GF 17 . Right panel: same plot but for the CO line in GF 20. The velocity resolutions are indicated in each panel. The vertical dashed lines separate the main core region from the filamentary region in each cloud. Note the systematic increase of the LSR velocities with right ascension offset away from position offsets $\Delta \alpha \sim 0^{\prime}$ in GF 17 and $\Delta \alpha \sim-8^{\prime}$ in GF 20.

depths. Although available for fewer positions, the $\mathrm{C}^{18} \mathrm{O}$ data was also used to probe the kinematic signatures that may exist within the denser regions.

In Fig. 15, the LSR central velocity of the ${ }^{13} \mathrm{CO}$ (GF 17) and $\mathrm{CO}$ (GF 20) lines is plotted against right ascension offset from the reference map positions. The velocity resolutions are $0.12 \mathrm{~km} \mathrm{~s}^{-1}$ for $\mathrm{CO}$ (in GF 17) and $0.11 \mathrm{~km} \mathrm{~s}^{-1}$ for ${ }^{13} \mathrm{CO}$ (in GF 20). The vertical dashed lines separate the main core region from the filamentary region in each cloud. A striking dependence (over $\sim 40^{\prime}$ in right ascension offset in both clouds) of line velocity with position is clearly seen. We focus our analysis on the following remarks:

1. We find a systematic, smooth increase of the LSR velocities away from position offsets $\Delta \alpha \sim 0^{\prime}$ in GF 17 and $\Delta \alpha \sim-8^{\prime}$ in GF 20 . These position offsets correspond to the lowest LSR velocities, respectively $V_{\mathrm{LSR}} \sim+3.9 \mathrm{~km} \mathrm{~s}^{-1}$ and $V_{\mathrm{LSR}} \sim+4.5 \mathrm{~km} \mathrm{~s}^{-1}$, observed toward GF 17 and GF 20, respectively. The line velocities in GF 17 increase smoothly to $V_{\mathrm{LSR}} \sim$ $+4.4 \mathrm{~km} \mathrm{~s}^{-1}$ (at $\left.\Delta \alpha \sim-10^{\prime}\right)$ toward the west, and to $V_{\mathrm{LSR}} \sim+4.3 \mathrm{~km} \mathrm{~s}^{-1}$ (at $\Delta \alpha \sim+30^{\prime}$ ) toward the east. For GF 20, the line velocities increase continuously to $V_{\mathrm{LSR}} \sim+5.2 \mathrm{~km} \mathrm{~s}^{-1}$ in both directions.
2. There appears to be an abrupt jump in $V_{\mathrm{LSR}}$ (from $\sim+4.1 \mathrm{~km} \mathrm{~s}^{-1}$ to $\sim+4.6 \mathrm{~km} \mathrm{~s}^{-1}$ ) occuring at the position offset $\Delta \alpha \sim+10^{\prime}$. This region corresponds to the dense core located just about $6^{\prime}$ to the northeast of the main core region seen in Fig. 1. We interpret this dense core as a dynamically single unit within GF 17 . However, note that this shift in velocity is smaller than the typical ${ }^{13} \mathrm{CO}$ line width in GF 17 , implying that if GF 17 was at a greater distance, it would be misinterpreted as a larger internal clump turbulence.

3. The dispersion in LSR velocities at each right ascension offset is generally larger in GF 17 than in GF 20. The filamentary region of GF 17 has larger velocity dispersions than the main core region, while the opposite is true for GF 20. In this cloud, the difference in velocity dispersions is partly due to the fact the lines are nearly gaussian in the filamentary region, but very assymetric in the main core region (owing to several blended velocity components along the line-of-sight, Moreira \& Yun 2002). For GF 17, the spectral lines toward the eastern half of main core region are made of two distinct, resolved velocity components, so that we have plotted in Fig. 15 only the stronger (blue-shifted) component. Also, note that the velocity dispersion observed at each right ascension offset is also a natural 
consequence of there being a dependence of the velocity gradient with declination offset.

4. In both clouds, the line velocities appear to become roughly constant (more evident in GF 20) at the eastern edge of the mapped region, while at the western edge $V_{\mathrm{LSR}}$ may well increase farther away to the west. In order to check if the velocity gradients could be traced outside our mapped regions, we have obtained additional $\mathrm{CO}$ and ${ }^{13} \mathrm{CO}$ spectra within GF 17 and GF 20, but outside the regions presented here. It turns out that $V_{\mathrm{LSR}} \sim+5 \mathrm{~km} \mathrm{~s}^{-1}$ at $\Delta \alpha \sim+75^{\prime}$, and $V_{\mathrm{LSR}} \sim+4.6 \mathrm{~km} \mathrm{~s}^{-1}$ at $\Delta \alpha \sim-25^{\prime}$ for GF 17 . Within GF 20 , we find $V_{\mathrm{LSR}} \sim+5.7 \mathrm{~km} \mathrm{~s}^{-1}$ at $\Delta \alpha \sim+40^{\prime}$ and $V_{\mathrm{LSR}} \sim+4.9 \mathrm{~km} \mathrm{~s}^{-1}$ at $\Delta \alpha \sim-30^{\prime}$. Thus, the trend of increasing velocity in GF 17 can be traced far beyond the mapped region, and in GF 20 the same trend can be traced to the east, while the LSR velocities appear to become more constant to the west.

5. The general behaviour depicted in Fig. 15 is well reproduced if we use instead the $\mathrm{C}^{18} \mathrm{O}$ maps of GF 17 and GF 20. This implies that our calculations of the velocity gradients are not significantly affected by opacity effects in the $\mathrm{CO}$ and ${ }^{13} \mathrm{CO}$ lines. The magnitude of the velocity gradients obtained here for GF 17 and GF 20 are typically 2 to 3 times larger than the previous estimates by Vilas-Boas et al. (2000).

From the considerations above, we conclude that the overall velocity gradient structure in GF 17 and GF 20 is remarkably similar (both morphologically and spatially), strongly suggesting a common origin. In order to investigate the origin of such velocity gradients, we need a quantitative analysis of the velocity structures of these clouds. We fit the $\mathrm{CO},{ }^{13} \mathrm{CO}$, and $\mathrm{C}^{18} \mathrm{O}$ maps of line-center velocity using a least-squares technique for the true direction and magnitude of the best-fit velocity gradient. The velocity, $V_{\mathrm{LSR}}$, at the peak of a symmetric emission profile is assumed to represent an intensity-weighted average velocity along the line of sight through the cloud.

If the cloud producing the emission line rotates as a solid body, $V_{\mathrm{LSR}}$ will be independent of distance along the line of sight, and linearly dependent on the coordinates in the plane of the sky (Goodman et al. 1993). Thus a cloud undergoing solid-body rotation can be expected to exhibit a linear gradient, $\nabla V_{\mathrm{LSR}}$, across the face of a map, perpendicular to the rotation axis. We fit the function $V_{\mathrm{LSR}}=V_{0}+a \Delta \alpha+b \Delta \delta$ to the data, where $\Delta \alpha$ and $\Delta \delta$ represent offsets in right ascension and declination, expressed in radians, $a$ and $b$ are the projections of the gradient per radian on the $\alpha$ and $\delta$ axes, and $V_{0}$ is the systemic velocity of the cloud, with respect to the local standard of rest. The magnitude of the velocity gradient, in a cloud at distance $D$, is then given by $\nabla V_{\mathrm{LSR}}=\left(a^{2}+b^{2}\right)^{1 / 2} / D$ and its direction (the direction of increasing velocity, measured east of north) is given by $\theta=\tan ^{-1}(a / b)$. From this, we can estimate the amount of solid-body rotation implied by the observed line of sight velocity field in a cloud. However, note that rotation is only one possible interpretation for any gradient found.

We have used the routine by Goodman et al. (1993), which performs a least-squares fit to the velocity field observed in a spectral-line map of a molecular cloud, and returns the magnitude of the gradient, its direction, and the errors in those quantities. Each observed value of $V_{\mathrm{LSR}}$ is weighted by $1 / \sigma_{V}^{2}$, where $\sigma_{V}$ is the uncertainty in $V_{\mathrm{LSR}}$ determined by a Gaussian fit to the line profile. Fitting all ${ }^{13} \mathrm{CO}$ data points in GF 17 , we find a best-fit velocity gradient of $\sim 0.46 \mathrm{~km} \mathrm{~s}^{-1} \mathrm{pc}^{-1}$ with direction $\theta=145^{\circ}$ east of north; fitting all the $\mathrm{C}^{18} \mathrm{O}$ data, one obtains the same magnitude for the best-fit velocity gradient and a slightly different direction, $\theta=154^{\circ}$. For GF 20 , the CO bestfit velocity gradient is $\sim 1.0 \mathrm{~km} \mathrm{~s}^{-1} \mathrm{pc}^{-1}$ with direction $\theta=134^{\circ}$, again in good agreement with the values derived from all the $\mathrm{C}^{18} \mathrm{O}$ data $\left(\nabla V_{\mathrm{LSR}}=0.76 \mathrm{~km} \mathrm{~s}^{-1} \mathrm{pc}^{-1}\right.$ and $\left.\theta=127^{\circ}\right)$. To estimate the significance of our derived velocity gradients, we calculate the ratio $\nabla V_{\mathrm{LSR}} / 3 \sigma$, where $\sigma$ is the error in the fitted velocity gradient. We find ratios of 267 and 16 for ${ }^{13} \mathrm{CO}$ and $\mathrm{C}^{18} \mathrm{O}$, respectively, in GF 17 , and 667 and 24 for $\mathrm{CO}$ and $\mathrm{C}^{18} \mathrm{O}$, respectively, in GF 20 . Thus, our best-fit velocity gradients are very robust.

Considering the plane-of-the-sky orientation of GF 20, we conclude that the overall velocity gradients found are roughly perpendicular to the major axis of the filamentary structure. In principle, this could be interpreted as largescale rotation of the cloud about its major axis. However, in this case a linear dependence of $V_{\mathrm{LSR}}$ along the full extent of the cloud would be expected. As shown in Fig. 15, this is not the case since the sense of the increase in $V_{\mathrm{LSR}}$ within the main core region does not coincide with that found for the filamentary region. To better analyze the kinematic differences between the filamentary regions and the main core regions of GF 17 and GF20, we have calculated best-fit velocity gradients within those regions separately. The results are listed in Table 2 . Clearly, the velocity gradients within the main core regions and those within the filamentary regions exhibit different plane-ofthe-sky orientations, but also differ in magnitude (typically a factor $2-2.5$ larger in the main core regions). Caution should be taken in interpreting the velocity shifts in the main core regions as simple streaming motions, because as mentioned above (1) these regions exhibit possibly several blended velocity components, and (2) the observed changes in velocity in those regions are comparable or smaller than the $\mathrm{CO}$ and ${ }^{13} \mathrm{CO}$ line widths therein, suggesting that we may be witnessing changes in the relative strength of different components along the line of sight. In any case, comparing the filamentary regions or the main core regions in both clouds, we find very similar magnitudes of the best-fit velocity gradients. We also note that the directions of the best-fit velocity gradients in the main core regions are remarkably similar. Taken together, these results argue that simple large-scale rotation cannot account for the velocity gradients and their similarities in GF 17 and GF 20, and instead strongly suggest a more complex, common origin. 
Table 2. Results of gradient fitting.

\begin{tabular}{|c|c|c|c|c|c|c|}
\hline Cloud & Region & $\begin{array}{c}\text { Gradient } \\
\left(\mathrm{km} \mathrm{s}^{-1} \mathrm{pc}^{-1}\right)\end{array}$ & $\begin{array}{c}\text { Direction } \\
(\operatorname{deg} \mathrm{E} \text { of } \mathrm{N})\end{array}$ & $\begin{array}{c}\text { Gradient } \\
\text { significance }\end{array}$ & $\begin{array}{l}\text { Number of } \\
\text { points in fit }\end{array}$ & $\begin{array}{c}\text { Region } \\
\text { of fit }\end{array}$ \\
\hline & & & & $\mathrm{CO}$ & & \\
\hline \multirow[t]{3}{*}{ GF 20} & filament & 0.48 & 40 & 180 & 397 & $\Delta \alpha>-8^{\prime}$ \\
\hline & main core & 1.22 & -83 & 392 & 132 & $\Delta \alpha<-8^{\prime}$ \\
\hline & & & & ${ }^{13} \mathrm{CO}$ & & \\
\hline \multirow[t]{3}{*}{ GF 17} & filament & 0.39 & 94 & 288 & 288 & $\Delta \alpha>0^{\prime}$ \\
\hline & main core & 0.80 & -80 & 47 & 80 & $\Delta \alpha<0^{\prime}$ \\
\hline & & & & $\mathrm{C}^{18} \mathrm{O}$ & & \\
\hline \multirow[t]{2}{*}{ GF 17} & filament & 0.66 & 63 & 23 & 108 & $\Delta \alpha>0^{\prime}$ \\
\hline & main core & 1.63 & -132 & 21 & 26 & $\Delta \alpha<0^{\prime}$ \\
\hline \multirow[t]{2}{*}{ GF 20} & filament & 0.30 & 153 & 7 & 264 & $\Delta \alpha>-8^{\prime}$ \\
\hline & main core & 2.29 & -157 & 9 & 9 & $\Delta \alpha<-8^{\prime}$ \\
\hline
\end{tabular}

Could galactic shear produce such velocity gradients? It seems very unlikely because the overall best-fit velocity gradients in GF 17 and GF 20 given above are nearly perpendicular to the galactic plane. Still, we can estimate the inclination to the plane of the sky of GF 17 and GF 20 which is required so that the velocity gradient induced by galactic shear reproduces the velocity gradients observed. The velocity gradient induced in a cloud with galactic longitude, $l$, at an inclination angle, $i$, to the plane-of-the-sky is given by $\Delta v=A \sin (2 l) \tan (i) \Delta r$, where $A$ is the Oort $A$ constant and $\Delta r$ is the offset position across the face of the cloud map. From Fig. 15 we take $\Delta v=0.8 \mathrm{~km} \mathrm{~s}^{-1}$, corresponding to the velocity shift observed across $\sim 27^{\prime}$ (or about $\Delta r=1.2 \mathrm{pc}$ ) along the filamentary regions of GF 17 or GF 20. Using $A=16 \mathrm{~km} \mathrm{~s}^{-1} \mathrm{kpc}^{-1}$ (Mihalas \& Binney 1981), we derive $i \sim 86^{\circ}$. Then, if galactic shear is the dominant mechanism producing the observed velocity gradient, this requires that GF 17 and GF 20 be nearly perpendicular to the plane-of-the-sky, i.e. along the lineof-sight. This seems very unlikely because it would imply that the true extent of the filamentary regions in GF 17 and GF 20 would have to be of the order of $\sim 17 \mathrm{pc}$, that is to say of the order of the diameter of the whole Lupus complex of dark clouds. Furthermore, these clouds appear to be small and dense, and thus insusceptible to the effects of a differential gravitational field. Thus, the velocity structures of GF 17 and GF 20 were likely produced by some mechanism other than galactic shear or simple largescale rotation. We discuss the nature of such mechanism in Sect. 5.2.

\section{Discussion}

\subsection{The source of dust heating}

The presence of extended far-infrared emission in these cold clouds poses several problems; first, what is the heating source for the dust in these clouds? Second, can a sufficient fraction of dust mass be heated to produce the observed intensity in the far-infrared? Both the absence of luminous embedded sources in these clouds and the observed gradient in dust temperature suggest that the dust heating must be external to these clouds. One possible source of heating is the low-luminosity stars which have formed within GF 17 and GF 20. However, the total luminosities of the populations of $\mathrm{T}$ Tauri stars associated with GF 17 and GF 20 amount to $\sim 4.6 L_{\odot}$ and $\sim 5.8 L_{\odot}$, respectively (Krautter 1991; Hughes et al. 1994). From the total luminosities calculated above we find that the T Tauri populations can only account for $20 \%$ of the farinfrared luminosity in GF 17 and GF 20. Thus, the young low-mass stars associated with GF 17 and GF 20 cannot be the dominant source of dust heating in these clouds.

A likely candidate for the heat source then is the interstellar radiation field (ISRF). Both Mathis et al. (1983) and de Muizon \& Rouan (1985) have computed models of interstellar clouds heated by a standard ISRF. Both studies found that the mean temperature of grains exposed to the standard ISRF is $10 \mathrm{~K}$ for silicate grains and $20 \mathrm{~K}$ for graphite grains (see also Draine \& Lee 1984), smaller than the average temperature of the grains responsible for the emission in our clouds. From the model calculations of Spencer \& Leung (1978), we find that the ratio of flux densities at 60 and $100 \mu \mathrm{m}$ should be approximately 0.55 for graphite grains and approximately 0.03 for silicate grains. The observed flux density ratio in our clouds range from 0.16 to 0.30 ; if the intensity of the ISRF is uniform, then our clouds must contain a mixture of the two types of grains. The fact that our calculated temperatures are significantly higher than $20 \mathrm{~K}$ indicates that if the ISRF is the dominant source of dust heating, then this ISRF has to be particularly intense near GF 17 and GF 20, as is the case in the $\rho$ Oph complex. 
This led us to investigate the possibility that the nearby Sco OB2 association is responsible for the high dust temperatures found within GF 17 and GF 20. Like the $\rho$ Oph cloud, the Lupus clouds lie close to the UpperScorpius (hereafter USco) subgroup $(D \sim 170 \mathrm{pc})$ of the Sco OB2 association (Murphy et al. 1986). The stars in USco that lie close to $\rho$ Oph contribute approximately $1000 L_{\odot}$ (Ryter et al. 1987; de Geus et al. 1989) by irradiating the cloud's surface (approximately $10 \mathrm{pc}^{2}$ ) with an absorbed flux of about $5 \times 10^{-2} \mathrm{erg} \mathrm{s}^{-1} \mathrm{~cm}^{-2}$. It is difficult to assess the three-dimensional configuration of GF 17, GF 20, and $\rho$ Oph relative to the USco subgroup. Still, we can make a rough estimate of the contribution of the OB2 association to the far-infrared luminosities of GF 17 and GF 20. The surface areas contained in our molecular maps of GF 17 and GF 20 are $0.32 \mathrm{pc}^{2}$ and $0.36 \mathrm{pc}^{2}$, respectively. Assuming that the true distance from the USco association to each one of the clouds is simply the projected distance on the plane of the sky, one finds that GF 20 and GF 17 are, respectively, roughly 2.5 and 3.3 times more distant to USco than the $\rho$ Oph cloud. This translates into absorbed fluxes of $\sim 4.6 \times 10^{-3}$ and $\sim 8.0 \times 10^{-3} \mathrm{erg} \mathrm{s}^{-1} \mathrm{~cm}^{-2}$ for GF 17 and GF 20, yielding far-infrared luminosities of $3.7 L_{\odot}$ and $7.2 L_{\odot}$. These values are in excellent agreement with the far-infrared luminosities derived above from our IRAS images, given the uncertainties. Therefore, contributions to the far-infrared luminosities of these clouds from unknown external or hidden internal sources must be minimal, and we conclude that the dominant source of dust heating in GF 17 and GF 20 is likely to be the ISRF due to the Sco OB2 association.

\subsection{The origin of the cloud structures}

The interaction between the $\rho$ Oph cloud complex and the USco association is well established (Olano \& Pöppel 1981; Cappa de Nicolau \& Pöppel 1986; Ryter et al. 1987; de Geus et al. 1989; Loren 1989; de Geus et al. 1990; Nozawa et al. 1991; de Geus 1992). The star formation efficiency (SFE) in $\rho$ Oph is very high $(\sim 20 \%)$, while it is only of the order of $0.3 \%$ in the Ophiuchus north region. Nozawa et al. (1991) suggested that such low SFE could be attributed to the high ionization degree due to the strong UV radiation field from the Sco OB2 association, which could prevent the molecular clouds therein from collapsing via strong coupling between magnetic fields and the molecular gas. More recently, Tachihara et al. (1996) found that the SFE in GF 20 is $\sim 0.9 \%$, while in Lupus $1(\sim 0.4 \%)$ it is comparable to that found by Nozawa et al. (1991) in the Ophiuchus north region. In Lupus 3, they found the SFE to be significantly higher $(\geq 3.8 \%)$. On the other hand, Hughes et al. (1994) showed that there is a large difference among the stellar ages associated with the dark clouds in Lupus. The T Tauri stars in Lupus 3 and GF 17 are on average much older (respectively $\sim 7 \times 10^{6} \mathrm{yr}$ and $\sim 4 \times 10^{6}$ yr $)$ than those in Lupus $1\left(\sim 8 \times 10^{5}\right.$ yr $)$ and GF $20\left(\sim 5 \times 10^{5}\right.$ yr $)$. Tachihara et al. (1996) interpreted these facts as an indication that star formation in Lupus 1 and GF 20 was triggered by the interaction with the giant HI expanding shell that surrounds the USco subgroup (Cappa de Nicolau \& Pöppel 1986; de Geus 1992). Figure 5 in Tachihara et al. (1996) shows that the Lupus complex of dark clouds is seen in projection against the edge of the USco shell, indicating that GF 17 and GF 20 are likely to be affected by the expanding shell.

This can also be seen in Fig. 16 were we plot the LSR velocities versus galactic latitude for every position in our molecular line maps of GF 17 and GF 20. Also plotted is the distribution of radial velocities from HI data (shown as crosses) from Heiles \& Habing (1974), Pöppel et al. (1979), and Cappa de Nicolau \& Pöppel (1986), collected both at the center $\left(0 \leq V_{\mathrm{lsr}} \leq 4 \mathrm{~km} \mathrm{~s}^{-1}\right)$ and edges $\left(V_{\mathrm{lsr}} \sim-4 \mathrm{~km} \mathrm{~s}^{-1}\right.$ and $\left.V_{\mathrm{lsr}} \sim+8 \mathrm{~km} \mathrm{~s}^{-1}\right)$ of the USco shell. The solid lines represent the best-fit model (Cappa de Nicolau \& Pöppel 1986) of an expanding shell with expansion velocity of $6 \mathrm{~km} \mathrm{~s}^{-1}$, for three different ratios of $R / r_{0}$, where the $R$ is the radius of the shell and $r_{0}$ is the distance to the observer. Also shown (dashed line) is a best-fit model by de Geus (1992) of a shell with radius $R=40 \mathrm{pc}$ and expanding at a larger velocity $\left(10 \mathrm{~km} \mathrm{~s}^{-1}\right)$, based on additional HI data in the USco and Ophiuchus regions from de Geus \& Burton (1991). Both models clearly show that GF 17 and GF 20 are within or at the boundaries of the expanding USco shell. On the other hand, considering the galactic coordinates of our clouds, we note that GF 17 and GF 20 are located near the Upper-Centaurus-Lupus (hereafter UCen-Lup) association, the oldest subgroup of the Sco OB2 association (see Fig. 1b in de Geus 1992). A best-fit model (de Geus 1992) of the expanding HI shell surrounding the UCen-Lup subgroup is also plotted (short-long dashed line) in Fig. 16. Much larger $(R \sim 110 \mathrm{pc})$ than the USco shell, its expanding velocity is $10 \mathrm{~km} \mathrm{~s}^{-1}$ with respect to its systemic velocity of $\sim 0 \mathrm{~km} \mathrm{~s}^{-1}$. We note that the UCen-Lup shell encompasses the USco shell. Then, at the current expansion rate the UCen-Lup shell must have passed the USco region. Therefore, it is likely that the Lupus complex of dark clouds (including GF 17 and GF 20) was also shaped by the UCen-Lup shell.

From the present-day expansion rate and radius of the HI shells, de Geus (1992) estimated upper limits for their dynamical time scale, and found $\sim 2.5 \times 10^{6}$ yr for the USco shell and $\sim 11 \times 10^{6}$ yr for the UCen-Lup shell. From the relative positions of GF 17 and GF 20 with respect to the UCen-Lup shell ( $\sim 42 \mathrm{pc}$ in the plane of the sky), we estimate that the shell must have passed the Lupus complex $\sim 7 \times 10^{6}$ yr ago. However, note that an inclination of $60^{\circ}$ to the plane of the sky can lower this estimate to about $\sim 3.5 \times 10^{6} \mathrm{yr}$, consistent with the estimate by de Geus (1992) that the UCen-Lup shell has passed the region of Upper-Scorpius about $4 \times 10^{6}$ yr ago. Thus, at the epoch when the USco shell was blown, the UCen-Lup shell was already passing through and compressing the Lupus clouds via the propagating shock front. From these numbers and the age of the $\mathrm{T}$ Tauri stars in the different 


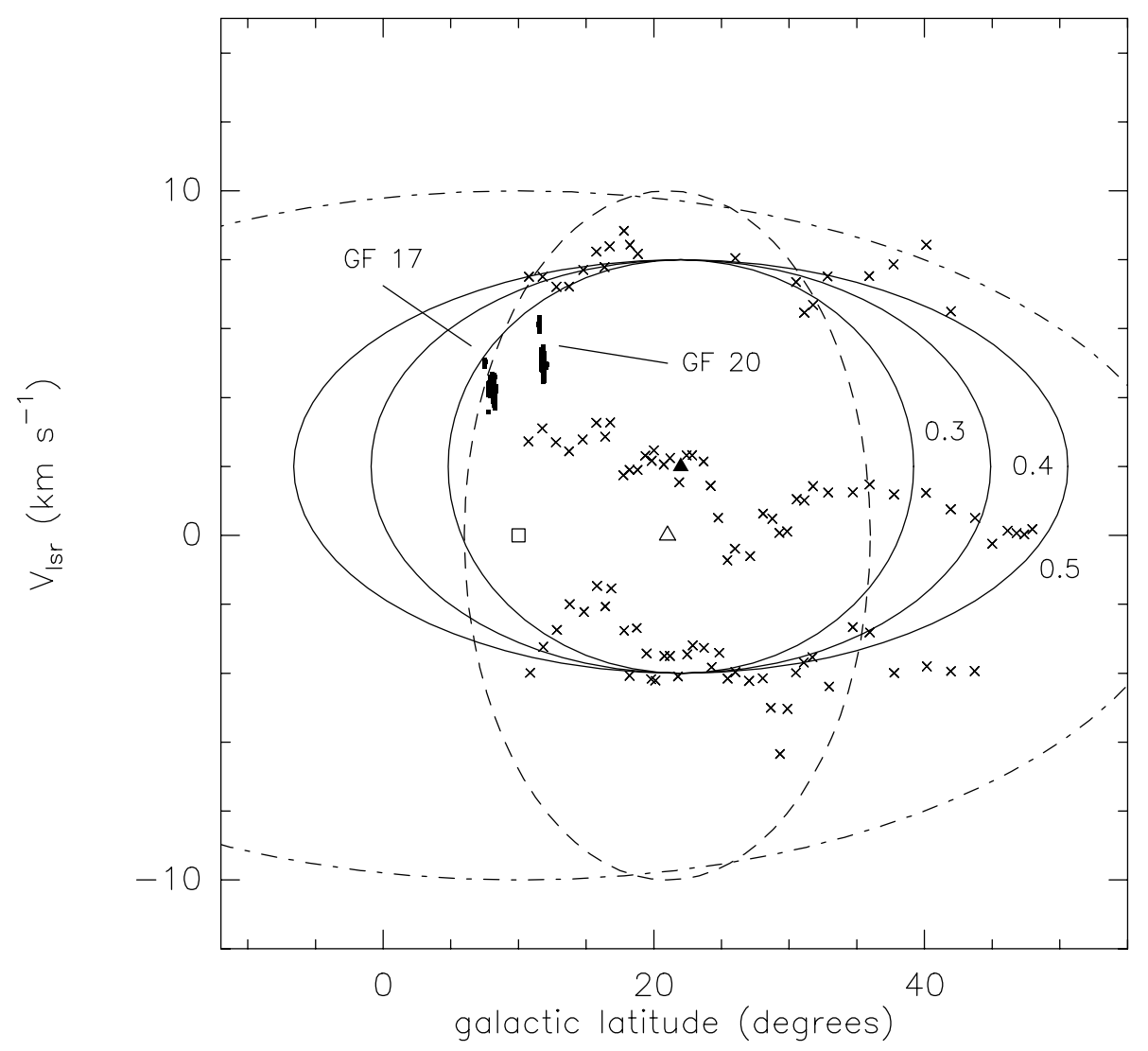

Fig. 16. Distribution of observed radial velocities in GF 17 and GF 20 versus galactic latitude (filled squares). The crosses represent the distribution of radial velocities from HI data from the Upper-Scorpius shell. The solid lines represent the bestfit models by Cappa de Nicolau \& Pöppel (1986) of an expanding shell with expansion velocity of $6 \mathrm{~km} \mathrm{~s}^{-1}$, centered at $(l, b)=\left(347^{\circ},+22^{\circ}\right)$, and with a systemic velocity of $2 \mathrm{~km} \mathrm{~s}^{-1}$ (shown by the filled triangle). The dashed line represents the best-fit model by de Geus (1992) of an expanding shell with expansion velocity of $10 \mathrm{~km} \mathrm{~s}^{-1}$, centered at $(l, b)=\left(347^{\circ},+21^{\circ}\right)$, and with a systemic velocity of $0 \mathrm{~km} \mathrm{~s}^{-1}$ (shown by the empty triangle). The short-long dashed line is the best-fit model by de Geus (1992) of the HI shell surrounding the Upper-Centaurus-Lupus subgroup, which expands at $10 \mathrm{~km} \mathrm{~s}$; the UpperCentaurus-Lupus shell is centered at $(l, b)=\left(320^{\circ},+10^{\circ}\right)$, has a systemic velocity of $\sim 1 \mathrm{~km} \mathrm{~s}^{-1}$ (shown by the empty square).

Lupus clouds given above, and from the median age of the T Tauri stars in Lupus $\left(\sim 3.2 \times 10^{6} \mathrm{yr}\right.$, Hughes et al. 1994), we conclude that it is possible that most of the $\mathrm{T}$ Tauri stars in Lupus 3 and GF 17 were formed due to the interaction with the UCen-Lup shell, and not with the USco shell. Apparently, at that time the Lupus 1 and GF 20 clouds were not dense enough to form stars since in these clouds almost all T Tauri stars are $1 \times 10^{6} \mathrm{yr}$ old or less (Hughes et al. 1994). The recent star formation in Lupus 1 and GF 20 was likely induced by the USco shell (Tachihara et al. 1996).

Can we find observational evidence that GF 17 and GF 20 were shaped by both shells, and not only by the USco shell as proposed by Tachihara et al. (1996)? In spite of the obvious difficulty in assessing the 3 dimensional configuration (including distances) of the Lupus clouds with respect to the expanding shells, this seems to be the case, if we take into consideration (1) the close correspondence between the age of the USco shell and the time when the UCen-Lup shell passed the Lupus region, and (2) the present-day morphology and gas kinematics of GF 17 and GF 20. The velocity gradients over the filamentary regions of GF 17 and GF 20 impose upper limits to the cloud crossing times, which we find to be $\sim 3 \times 10^{6}$ yr and $\sim 2 \times 10^{6}$ yr, respectively, consistent with the time scales given above.

Moreover, the $\mathrm{C}^{18} \mathrm{O}$ velocity gradient observed along the filamentary region of GF 17 is aligned (within $5^{\circ}$ ) with the direction to, and away from the center of the UCen-Lup shell. Also, the CO velocity gradient in the filamentary region of GF 20 is also aligned, within $5^{\circ}$, with the direction toward the UCen-Lup shell, and points away from the shell. From a morphological point of view, GF 17 (which extends well beyond the region studied here) is closely aligned with the direction pointing toward the center of the UCen-Lup shell, and a similar behaviour is seen for GF 20; this is also the case of the Lupus 3 cloud (Tachihara et al. 1996). Finally, we note that the sense of increasing velocity (inferred from the positions of GF 17 and GF 20 in the LSR velocity-galactic latitude diagram in Fig. 16, which are located symmetrically with respect to the semi-minor velocity axis of the UCen-Lup shell) follows reasonably well the directions pointing away from the center (shown as an empty square) of the UCen-Lup shell, 
and not from the center of the USco shell, for either model shown. Thus, it seems plausible that the velocity gradients along the filamentary regions of GF 17 and GF 20 were originated by the interaction with the UCen-Lup shell.

On the other hand, considering the galactic coordinates of GF 20, we point out that the elongated main core region south of the $\mathrm{T}$ Tauri star RU Lupi points away from the center of the USco shell. Considering all positions within GF 17 and GF 20, the best-fit velocity gradients point away (within $20^{\circ}$ ) from the center of the USco shell. If the velocity gradients found in the main core regions of GF 17 and GF 20 represent bona fide large-scale streaming motions, their dynamical time scale is typically smaller than their counterparts in the filamentary regions by a factor of 2 , suggesting that perhaps they did not originate from the interaction with the UCen-Lup shell, but instead were produced by the interaction with the more recent USco shell event. Taken together this facts seem to indicate that the swept-up appearance of GF 17 and GF 20 is likely due to the interaction with both the UCen-Lup and the USco shells, and not the USco shell alone.

\section{Summary}

In this paper we present IRAS co-added images and large-scale $\mathrm{CO}(1-0),{ }^{13} \mathrm{CO}(1-0)$, and $\mathrm{C}^{18} \mathrm{O}(1-0)$ millimeter molecular line observations of the globular filaments GF 17 and GF 20 in the Lupus complex of dark clouds. The IRAS images have revealed that these clouds have extended emission at 60 and $100 \mu \mathrm{m}$. We have used a simple uniform dust excitation model to derive opacities, color temperatures, and visual extinctions for the dust emitting at 60 and $100 \mu \mathrm{m}$. We derived gas column densities, have searched for correlations between gas and dust, and analysed the velocity structure of GF 17 and GF 20. We summarize our findings below:

- We find a good agreement between the $100 \mu \mathrm{m}$ optical depth images and the ${ }^{13} \mathrm{CO}$ integrated emission maps. The dust opacity is well correlated with ${ }^{13} \mathrm{CO}$ integrated intensity within the filamentary regions of GF 17 and GF 20. However, no correlation between these two quantities is found within the denser main core region of GF 17, probably due to saturation of the ${ }^{13} \mathrm{CO}$ emission therein. Comparison of the $100 \mu \mathrm{m}$ optical depth with $\mathrm{C}^{18} \mathrm{O}$ integrated intensity in GF 17 and GF 20 indicates that the dust optical depth and the gas column density are well correlated.

- The variation of the dust color temperature with position in GF 17 and GF 20 clearly requires the presence of an external dust heating mechanism capable of penetrating these clouds to a reasonable depth. The best candidate for the external source heating the dust in GF 17 and GF 20 is the ISRF due to the nearby Sco OB2 association.

- We find a correlation between the measured dispersion in our extinction determinations and the extinction toward both clouds, which is very similar to that found for the L977 and IC 5146 clouds, and interpret this as evidence that the edges of GF 17 and GF 20 are characterized by a smooth density gradient.

- Analysis of the gas velocity structure within GF 17 and GF 20 reveals evidence for smooth large-scale streaming motions along the filamentary structures with magnitude $\sim 0.5 \mathrm{~km} \mathrm{~s}^{-1} \mathrm{pc}^{-1}$. These velocity gradients cannot be interpreted easily as large-scale rotation or shear. Instead, we argue that the smooth velocity gradients observed over the full extents of GF 17 and GF 20 are the best evidence that the gas kinematics in these clouds is likely due to a propagating shock front. Both the dynamical time scales (a few million years) and the spatial orientation of these velocity gradients provide observational evidence for interactions of the GF 17 and GF 20 globular filaments with the Upper-Scorpius and Upper-CentaurusLupus HI expanding shells.

Acknowledgements. The authors would like to thank Dr. Dan Clemens for providing the co-added IRAS images, and the SEST operators Felipe McAuliffe and Francisco Azagra for assistance with the observations. This work has been partially supported by a grant from Fundação para a Ciência e Tecnologia (FCT, Portugal) to J.L.Y. Support from FCT to M.C.M. in the form of a scholarship is gratefully acknowledged.

\section{References}

Alves, J., Lada, C. J., Lada, E. A., Kenyon, S. J., \& Phelps, R. 1998, ApJ, 506, 292

Andreazza, C. M., \& Vilas-Boas, J. W. S. 1996, A\&AS, 116, 21

Arce, H. G., \& Goodman, A. A. 1999, ApJ, 517, 264

Beckwith, S. V. W., \& Sargent, A. I. 1991, ApJ, 381, 250

Blitz, L., Magnani, L., \& Mundy, L. 1984, ApJ, 282, L9

Booth, R. S., Delgado, G., Hagstrom, M., et al. 1989, A\&A, 216,315

Boulanger, F., \& Perault, M. 1988, ApJ, 330, 964

Boulanger, F., Baud, B., \& van Albada, G. D. 1985, A\&A, 144, L9

Boulanger, F. 1989, in The Physics and Chemistry of Interstellar Molecular Clouds - $\mathrm{mm}$ and Sub-mm Observations in Astrophysics, Proc. of the Symp., Zermatt, Switzerland (Springer-Verlag), 30

Bourke, T. L., Garay, G., Lehtinen, K. K., et al. 1997, ApJ, 476, 781

Cappa de Nicolau, C. E., \& Pöppel, W. G. L. 1986, A\&A, 164, 274

Clemens, D. P., Yun, J. L., \& Heyer, M. H. 1991, ApJS, 75, 877

Draine, B. T., \& Lee, H. M. 1984, ApJ, 285, 89

Draine, B. T. 1990, in The Interstellar Medium in Galaxies, ed. H. A. Thronson Jr, \& J. M. Shull (Dordrecht: Kluwer), 483

Frerking, M. A., Langer, W. D., \& Wilson, R. L. 1982, ApJ, 262,590

Gahm, G. F., Johansson, L. E. B., \& Liseau, R. 1993, A\&A, 274,415

de Geus, E. J., Zeeuw, P. T., \& Lub, J. 1989, A\&A, 216, 44

de Geus, E. J., Bronfman, L., \& Thaddeus, P. 1990, A\&A, 231, 137

de Geus, E. J., \& Burton, W. B. 1991, A\&A, 246, 559

de Geus, E. J. 1992, A\&A, 262, 258 
Goodman, A. A., Benson, P. J., Fuller, G. A., \& Myers, P. C. 1993, ApJ, 406, 528

Heiles, C., \& Habing, H. J. 1974, A\&AS, 14, 1

Heiles, C. 1997, ApJS, 111, 245

Hildebrand, R. H. 1983, Quart. J. R. A. S., 24, 267

Hughes, J., Hartigan, P., Krautter, J., \& Kelemen, J. 1994, AJ, 108, 1071

Humphreys, R. M. 1978, ApJS, 38, 309

Jarrett, T. H., Dickamn, R. L., \& Herbst, W. 1989, ApJ, 345, 881

Krautter, J. 1991, in Low Mass Star Formation in Southern Molecular Clouds, ed. B. Reipurth, ESO Scientific Rep., 11, 127

Krautter, J., Wichmann, R., Schmitt, J. H. M. M., et al. 1997, A\&AS, 123, 329

Kutner, M. L., \& Ülich, B. L. 1981, ApJ, 250, 341

Lada, C. J., Lada, E. A., Clemens, D. P., \& Bally, J. 1994, ApJ, 429, 694

Lada, C. J., Alves, J., \& Lada, E. A. 1999, ApJ, 512, 250

Langer, W. D., Wilson, R. W., Goldsmith, P. F., \& Beichman, C. A. 1989, ApJ, 337, 355

Leung, C. M. 1985, in Protostars and Planets II, ed. D. C. Black, \& M. S. Matthews (Tucson: UA Press), 104

Loren, R. B. 1989, ApJ, 338, 925

Low, F. J., Young, E., Beintena, D. A., et al. 1984, ApJ, 278, L19

Mannings, V., \& Emerson, J. P. 1994, MNRAS, 267, 361

Mathis, J. S., Mezger, P. G., \& Panagia, N. 1983, A\&A, 128, 212

Mihalas, D., \& Binney, J. 1981, in Galactic astronomy: Structure and kinematics, 2nd edition, ed. W. H. Freeman, \& Co. (San Francisco, CA), 478

Moreira, M. C., Jessop, N. E., Santos, C. A., \& Yun, J. L. 2000, AJ, 119, 2960

Moreira, M. C., \& Yun, J. L. 2002, in preparation de Muizon, J. M., \& Rouan, D. 1985, A\&A, 143, 1601

Murphy, D. C., Cohen, R., \& May, J. 1986, A\&A, 167, 234

Nozawa, S., Mizuno, A., Teshima, Y., Ogawa, H., \& Fukui, Y. 1991, ApJ, 77, 647
Nyman, L. A., \& Booth 1990, communication at the 29th Liège Colloq., ESA SP-314

Olano, C. A., \& Pöppel, W. G. L. 1981, A\&A, 95, 316

Padoan, P., Jones, B. J. T., \& Nordlund, Å. P. 1997, ApJ, 474, 730

Pollack, J. B., Hollenbach, D., Simonelli, D. P., Roush, T., \& Fong, W. 1994, ApJ, 421, 615

Pöppel, W. G. L., Vieira, E. R., Olano, C. A., \& Franco, M. L. 1979, First Latin-American Regional Astronomy Meeting, ed. A. Gutiérrez Moreno, \& H. Moreno (Santiago de Chile), 188

Puget, J. L., \& Léger, A. 1989, ARA\&A, 27, 161

Reipurth, B. 1994, A general catalog of Herbig-Haro objects, electronically published via anonymous ftp to ftp.hq.eso.org, directory /pub/Catalogs/Herbig-Haro

Ryter, C., Puget, J. L., \& Perault, M. 1987, A\&A, 186, 312

Schneider, S., \& Elmegreen, B. G. 1979, ApJS, 41, 87

Schwartz, R. D. 1977, ApJS, 35, 161

Scoville, N. Z., \& Good J. C. 1987, in Star Formation in Galaxies, Proc. of a NASA meeting, 3

Snell, R. L., Heyer, M. H., \& Schloerb, F. P. 1989, ApJ, 337, 739

Spencer, R. G., \& Leung, C. M. 1978, ApJ, 222, 140

Tachihara, K., Dobashi, K., Mizuno, A., Ogawa, H., \& Fukui, Y. 1996, PASJ, 48, 489

Terebey, S., \& Fich, M. 1986, ApJ, 309, L73

Thoraval, S., Boissé, P., \& Duvert, G. 1997, A\&A, 319, 948

Vilas-Boas, J. W. S., Myers, P. C., \& Fuller, G. A. 2000, ApJ, 532,1038

Vrba, F. J. 1977, AJ, 82, 198

de Vries, H. w., Heithausen, A., \& Thaddeus, P. 1987, ApJ, 319,723

Weiland, J. L., Blitz, L., Dwek, E., et al. 1986, ApJ, 306, L101

Weintraub, D. A., Sandell, G., \& Duncan, W. D. 1991, ApJ, 382,270

Wichmann, R., Krautter, J., Covino, E., et al. 1997, A\&A, 320, 185

Wood, D. O. S., Myers, P. C., \& Daugherty, D. A. 1994, ApJS, 95,457 